\title{
GRB Fermi-LAT Afterglows: Explaining Flares, Breaks, and Energetic Photons
}

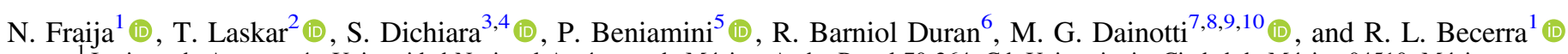 \\ ${ }^{1}$ Instituto de Astronomía, Universidad Nacional Autónoma de México, Apdo. Postal 70-264, Cd. Universitaria, Ciudad de México 04510, México; \\ nifraija@astro.unam.mx \\ ${ }^{2}$ Department of Physics, University of Bath, Claverton Down, Bath, BA2 7AY, UK \\ ${ }^{3}$ Department of Astronomy, University of Maryland, College Park, MD 20742-4111, USA \\ ${ }^{4}$ Astrophysics Science Division, NASA Goddard Space Flight Center, 8800 Greenbelt Road, Greenbelt, MD 20771, USA \\ 5 TAPIR, Mailcode 350-17, California Institute of Technology, Pasadena, CA 91125, USA \\ ${ }^{6}$ Department of Physics and Astronomy, California State University, Sacramento, 6000 J Street, Sacramento, CA 95819-6041, USA \\ ${ }^{7}$ Physics Department, Stanford University, 382 Via Pueblo Mall, Stanford, USA \\ ${ }^{8}$ Space Science Institute, Boulder, Colorado, USA \\ ${ }^{9}$ Obserwatorium Astronomiczne, Uniwersytet Jagielloński, ul. Orla 171, 31-501 Kraków, Poland \\ ${ }^{10}$ Interdisciplinary Theoretical \& Mathematical Science Program, RIKEN (iTHEMS), 2-1 Hirosawa, Wako, Saitama 351-0198, Japan \\ Received 2020 June 15; revised 2020 October 12; accepted 2020 October 19; published 2020 December 18
}

\begin{abstract}
The Fermi-LAT collaboration presented the second gamma-ray burst (GRB) catalog covering its first 10 years of operations. A significant fraction of afterglow-phase light curves in this catalog cannot be explained by the closure relations of the standard synchrotron forward-shock model, suggesting that there could be an important contribution from another process. In view of the above, we derive the synchrotron self-Compton (SSC) light curves from the reverse shock in the thick- and thin-shell regime for a uniform-density medium. We show that this emission could explain the GeV flares exhibited in some LAT light curves. Additionally, we demonstrate that the passage of the forward shock synchrotron cooling break through the LAT band from jets expanding in a uniformdensity environment may be responsible for the late time $\left(\approx 10^{2} \mathrm{~s}\right)$ steepening of LAT GRB afterglow light curves. As a particular case, we model the LAT light curve of GRB 160509A that exhibited a GeV flare together with a break in the long-lasting emission, and also two very high energy photons with energies of 51.9 and $41.5 \mathrm{GeV}$ observed 76.5 and $242 \mathrm{~s}$ after the onset of the burst, respectively. Constraining the microphysical parameters and the circumburst density from the afterglow observations, we show that the GeV flare is consistent with an SSC reverse-shock model, the break in the long-lasting emission with the passage of the synchrotron cooling break through the Fermi-LAT band, and the very energetic photons with SSC emission from the forward shock, when the outflow carries a significant magnetic field $\left(R_{\mathrm{B}} \simeq 30\right)$ and it decelerates in a uniform-density medium with a very low density $\left(n=4.554_{-1.121}^{+1.128} \times 10^{-4} \mathrm{~cm}^{-3}\right)$.
\end{abstract}

Unified Astronomy Thesaurus concepts: Gamma-ray bursts (629); Radiative processes (2055); Magnetic fields (994); Non-thermal radiation sources (1119); Interstellar medium (847)

\section{Introduction}

Gamma-ray bursts (GRBs) are the most energetic astrophysical sources in the universe. These events exhibit intense and nonuniform gamma-ray flashes created by inhomogeneities within the ultrarelativistic outflows (Zhang \& Mészáros 2004). The temporal and spectral features inferred from the early and late emissions, usually known as prompt-to-afterglow, respectively, can be interpreted within the context of the fireball model (e.g., see Cavallo \& Rees 1978). This involves a relativistic outflow that moves into the circumstellar medium and generates an outgoing (forward) shock and a reverse shock (RS) that propagates back into the outflow. Electrons accelerated in the forward shock (FS), and RS are cooled down principally by synchrotron and synchrotron-self Compton (SSC) emission. The synchrotron emission from the FS region is predicted to produce long-lasting afterglow emission (Sari et al. 1998) that can extend to $>100 \mathrm{MeV}$ energies (Kumar \& Barniol Duran 2009, 2010). Synchrotron emission from the RS is expected to generate an optical flash or an X-ray flare (Kobayashi 2000; Kobayashi \& Zhang 2007; Kobayashi et al. 2007; Fraija \& Veres 2018; Becerra et al. 2019; Ayache et al. 2020). If no new electrons are injected, then the material at the RS cools adiabatically. Synchrotron photons at the RS region can be upscattered by the same electron population in order to describe the gamma-ray flares (Kobayashi \& Zhang 2007; Fraija 2015; Fraija et al. 2016a, 2016b). The prompt-to-afterglow transition is usually observed in the X-ray light curves as a steep decay interpreted as high-latitude emission (Kumar \& Panaitescu 2000; Nousek et al. 2006; Willingale et al. 2010), or in some cases, as the X-ray flares/ optical flashes described in terms of the synchrotron radiation and SSC emission from an early afterglow (e.g., see Zhang et al. 2003; Kobayashi \& Zhang 2003; Zhang \& Kobayashi 2005; Kobayashi \& Zhang 2007).

Recently, Ajello et al. (2019) presented the second FermiLAT (Large Area Telescope) GRB catalog, covering the first 10 years of operations (from 2008 to 2018 August 4). The catalog comprises 169 GRBs with high-energy emission at $\geqslant 100 \mathrm{MeV}$, including 29 GRBs with prompt emission extending to $\geqslant 10 \mathrm{GeV}$. A large number (86) of LAT GRBs exhibited temporally extended (hereafter called "long-lasting") emission. A subset of these events (21 GRBs) exhibited a break in the LAT light curve between 63 and $1250 \mathrm{~s}$. Although the long-lasting emission is usually interpreted as synchrotron radiation from external FSs (Kumar \& Barniol Duran 2009, 2010), not all the LAT light curves satisfy the relation between power-law (PL) temporal and spectral indices or the closure relations that are expected in case the FS dominates the emission (Sari et al. 1998). Furthermore, there is 
evidence that the entirety of the $\mathrm{GeV}$ light curve for several bursts cannot be adequately explained as originating in FS synchrotron radiation alone (Maxham et al. 2011). In addition, half the bursts with long-lasting LAT emission exhibit light curves that peak before the prompt emission ends.

In bursts with high isotropic-equivalent prompt $\gamma$-ray energy $\left(E_{\text {iso }} \gtrsim 10^{53} \mathrm{erg}\right.$ ), such as GRB 080916C, GRB 090510A, GRB 090902B, GRB 090926A, GRB 110731A, and GRB 130427A (e.g., see Abdo et al. 2009a; ,2009b Ackermann 2010; Ackermann et al. 2011; Ackermann 2013; Ackermann et al. 2014), the LAT light curves exhibited a short and bright peak (hereafter called a GeV flare). For instance, GRB 160509A exhibited a GeV flare peaking at $\sim 20 \mathrm{~s}$, followed by a longlasting emission with a break at $\sim 300 \mathrm{~s}$. This burst exhibited the second highest-energy photon reported by the second Fermi-LAT GRB catalog, a $52 \mathrm{GeV}$ event that arrived $77 \mathrm{~s}$ after the onset of the burst.

To interpret $\mathrm{GeV}$ flares in the LAT light curves, we derive the SSC light curves from the RS. We also investigate the break exhibited at hundreds of seconds using the synchrotron FS model. As a particular case of the thick-shell regime, we describe the LAT observations (the GeV flare and the break in the long-lasting emission) of GRB 160509A and constrain the model parameters from the afterglow observations. We interpret the very energetic photons detected in the SSC framework from the FS. As an example of the thin-shell regime, we calculate the SSC emission from the optical flash displayed in GRB 180418A.

The paper is organized as follows. In Section 2, we derive the SSC light curves from the RS in the thick- and thin-shell regime and analyze the break exhibited in the long-lasting emission in the synchrotron FS framework. In Section 3, we model the LAT observations of GRB 160509A and discuss the implications of the results. In Section 4, we estimate the SSC light curves in the thin-shell regime for GRB 180418A, and in Section 5, we summarize. The convention $Q_{\mathrm{x}}=Q / 10^{\mathrm{x}}$ in c.g. s. units will be adopted throughout this paper.

\section{Connection between Theory and Fermi LAT Observations}

\subsection{SSC Light Curves from $R S$}

An RS propagating into the GRB jet (a "shell") is produced when the relativistic ejecta interacts with the external medium (Mészáros \& Rees 1997; Sari \& Piran 1999; Kobayashi \& Sari 2000). The dynamics of the RS can be described through the Sedov length (Sari \& Piran 1995)

$$
\ell=\left(\frac{3 E_{\mathrm{K}}}{4 \pi m_{p} c^{2} n}\right)^{\frac{1}{3}},
$$

and the bulk Lorentz factor during the deceleration phase

$$
\begin{aligned}
\Gamma & =\left(\frac{\ell}{\Delta}\right)^{\frac{3}{8}}, \\
& =\left(\frac{3 E_{\mathrm{K}}(1+z)^{3}}{32 \pi m_{p} c^{5} n}\right)^{\frac{1}{8}} t_{\mathrm{x}}^{-\frac{3}{8}},
\end{aligned}
$$

where $\Delta=2 c(1+z)^{-1} t_{\mathrm{x}}$ is the observed width of the shell given by the RS crossing time $t_{\mathrm{x}}, n$ is the density of the circumburst medium (assumed uniform), $E_{\mathrm{K}}$ is the isotropic equivalent kinetic energy, $c$ is the speed of light, and $m_{p}$ is the proton mass. Taking into account the duration of the burst $\left(T_{90}\right.$ ${ }^{11}$ ), it is possible to define two regimes: the thick-shell regime for $\Gamma_{c}<\Gamma$ (corresponding to $t_{\mathrm{x}} \lesssim T_{90}$ ) and the thin-shell regime for $\Gamma<\Gamma_{c}\left(T_{90}<t_{\mathrm{x}}\right)$, where the critical Lorentz factor is defined by

$$
\Gamma_{c} \equiv\left(\frac{3 E_{\mathrm{K}}(1+z)^{3}}{32 \pi m_{p} c^{5} n}\right)^{\frac{1}{8}} T_{90}^{-\frac{3}{8}}
$$

The RS accelerates electrons in the shell to relativistic energies, which radiate photons via synchrotron and SSC processes. The synchrotron process has been widely explored and discussed in Kobayashi (2000). We expand the established RS synchrotron formalism to include the expected contribution of SSC emission in a uniform-density environment in both thick- and thin-shell limits.

\subsubsection{The Thick-shell Regime $\left(\Gamma_{c}<\Gamma\right)$}

In the thick-shell regime, the RS becomes ultrarelativistic and therefore can decelerate the shell substantially. In this case, the shock crossing time is less than the duration of the burst $\left(t_{\mathrm{x}} \lesssim T_{90}\right)$. To derive the SSC light curves for the thick-shell regime, we describe the dynamics before and after the shock crossing time, separately.

2.1.1.1. Before the shock crossing time $\left(t<t_{\mathrm{x}}\right)$ At $t<t_{\mathrm{x}}$, the Lorentz factor of the fluid behind the RS evolves as $\gamma_{3} \propto t^{-1 / 4}$ due to adiabatic expansion, ${ }^{12}$ where $t$ is the lab-frame time. During this period and in the same region, the post-shock magnetic field ${ }^{13} B^{\prime} \propto t^{-1 / 4}$, and the Lorentz factor of the lowest-energy electrons $\gamma_{\mathrm{m}, \mathrm{r}} \propto t^{1 / 4}$. Electrons above $\gamma_{\mathrm{c}, \mathrm{r}} \propto t^{-1 / 4}$ cool efficiently via synchrotron emission. The corresponding synchrotron break frequencies at the RS region evolve as $\nu_{\mathrm{m}, \mathrm{r}}^{\mathrm{syn}} \propto t^{0}$ and $\nu_{\mathrm{c}, \mathrm{r}}^{\mathrm{syn}} \propto t^{-1}$, while the spectral peak flux density $F_{\text {max,r }}^{\text {syn }} \propto t^{\frac{1}{2}}$ (Kobayashi 2000). Hereafter, the subindex " $r$ " refers to the derived quantities in the RS.

Electrons accelerated by the RS can upscatter synchrotron photons, yielding an SSC spectrum characterized by the break frequencies $h \nu_{\mathrm{i}, \mathrm{r}}^{\mathrm{ssc}} \sim \gamma_{\mathrm{i}, \mathrm{r}}^{2} h \nu_{\mathrm{i}, \mathrm{r}}^{\mathrm{syn}}$, with $\mathrm{i}=\mathrm{m}$ and c. The flux of $\mathrm{SSC}$ emission can be computed as $F_{\max }^{\mathrm{ssc}} \sim \frac{4}{3} \frac{p-2}{p-1} \sigma_{T} n R F_{\max }^{\mathrm{syn}}$, where $R$ is the deceleration radius and $\sigma_{T}$ the Thompson cross section (Kobayashi 2000).

\footnotetext{
11 The choice of $T_{90}$ can be indicative of the duration of the burst, but this is not the only viable choice (Willingale et al. 2007; Dainotti et al. 2011; Ajello et al. 2019).

12 During RS crossing, the RS and FS divide the system into four regions: (1) unshocked ISM, (2) shocked ISM, (3) shocked ejecta, and (4) unshocked ejecta, respectively.

${ }^{13}$ Primes correspond to the quantities in a comoving frame.
} 
Based on the foregoing, the spectral breaks and the maximum flux of SSC emission can be written as ${ }^{14}$

$$
\begin{aligned}
h \nu_{\mathrm{m}, \mathrm{r}}^{\mathrm{ssc}} \simeq & 6.1 \times 10^{2} \mathrm{eV}(1+z)^{-1} \epsilon_{\mathrm{e}_{\mathrm{r}},-1}^{4} \epsilon_{\mathrm{B}_{\mathrm{r}},-1}^{\frac{1}{2}} n_{-4}^{\frac{3}{4}} \Gamma_{3}^{4} \Delta_{12}^{\frac{1}{4}} \\
& \times E_{\mathrm{K}, 53}^{-\frac{1}{4}} t_{1}^{\frac{1}{2}}, \\
h \nu_{\mathrm{c}, \mathrm{r}}^{\mathrm{ssc}} \simeq & 2.1 \times 10^{3} \mathrm{TeV}(1+z)^{3}\left(1+Y_{\mathrm{r}}\right)^{-4} \\
& \times \epsilon_{\mathrm{B}_{\mathrm{r}},-1}^{-\frac{7}{2}} n_{-4}^{-\frac{9}{4}} \Delta_{12}^{\frac{5}{4}} E_{\mathrm{K}, 53}^{-\frac{5}{4}} \times t_{1}^{-\frac{3}{2}}, \\
F_{\mathrm{max}, \mathrm{r}}^{\mathrm{ssc}} \simeq & 3.6 \times 10^{-6} \mathrm{mJy} \epsilon_{\mathrm{B}_{\mathrm{r}},-1}^{\frac{1}{2}} n_{-4} \Gamma_{3}^{-1} \Delta_{12}^{-\frac{3}{2}} d_{\mathrm{z}, 28}^{-2} E_{\mathrm{K}, 53}^{\frac{3}{2}} t_{1},
\end{aligned}
$$

where $d_{\mathrm{z}}$ is the luminosity distance and the value of $\Gamma$ is the bulk Lorentz factor estimated with Equation (2). Hereafter, we use $\frac{p-2}{p-1} \simeq 0.29$ with $p=2.4$ the spectral index.

The Klein-Nishina (KN) suppression effect must be considered in SSC emission due to the reduction of the emissivity compared with the classical Compton regime. In the $\mathrm{KN}$ regime, the location of the RS SSC cooling break is given by Fraija et al. (2019a)

$$
h \nu_{\mathrm{c}, \mathrm{r}}^{\mathrm{KN}} \simeq 40.9 \mathrm{TeV}\left(1+Y_{\mathrm{r}}\right)^{-1} \epsilon_{\mathrm{B}_{\mathrm{r}},-1}^{-1} n_{-4}^{-\frac{3}{4}} E_{K, 53}^{-\frac{1}{4}} \Delta_{12}^{\frac{1}{4}} t_{1}^{-\frac{1}{2}},
$$

where $Y_{\mathrm{r}}$ is the Compton parameter for the RS due to the KN effect (Wang et al. 2010; Beniamini et al. 2015; Fraija et al. 2019a).

Taking into account the electron Lorentz factors, the spectral breaks and the maximum flux, the SSC light curves for the fastand slow-cooling regime are

$$
F_{\nu, r}^{\mathrm{ssc}} \propto \begin{cases}t^{\frac{3}{2}} \nu^{\frac{1}{3}}, & \nu<\nu_{\mathrm{c}, \mathrm{r}}^{\mathrm{ssc}}, \\ t^{\frac{1}{4}} \nu^{-\frac{1}{2}}, & \nu_{\mathrm{c}, \mathrm{r}}^{\mathrm{ssc}}<\nu<\nu_{\mathrm{m}, \mathrm{r}}^{\mathrm{ssc}}, \\ t^{\frac{p}{4}} \nu^{-\frac{p}{2}}, & \nu_{\mathrm{m}, \mathrm{r}}^{\mathrm{ssc}}<\nu,\end{cases}
$$

and

$$
F_{\nu, r}^{\mathrm{ssc}} \propto \begin{cases}t^{\frac{5}{6}} \nu^{\frac{1}{3}}, & \nu<\nu_{\mathrm{m}, \mathrm{r}}^{\mathrm{ssc}}, \\ t^{\frac{p+3}{4}} \nu^{-\frac{p-1}{2}}, & \nu_{\mathrm{m}, \mathrm{r}}^{\mathrm{ssc}}<\nu<\nu_{\mathrm{c}, \mathrm{r}}^{\mathrm{ssc}}, \\ t^{\frac{p}{4}} \nu^{-\frac{p}{2}}, & \nu_{\mathrm{c}, \mathrm{r}}^{\mathrm{ssc}}<\nu,\end{cases}
$$

respectively, where $\nu$ is the observed frequency at a given time $t$.

2.1.1.2. After the shock crossing time $\left(t>t_{\mathrm{x}}\right)$ During this period, the post-shock magnetic field evolves as $B^{\prime} \propto t^{-\frac{13}{24}}$, and the Lorentz factor of the lowest-energy electrons $\gamma_{\mathrm{m}, \mathrm{r}} \propto t^{-\frac{13}{48}}$. Electrons above $\gamma_{\mathrm{c}, \mathrm{r}} \propto \gamma_{3}^{-1} B^{-2} t^{-1} \propto t^{\frac{3}{2}}$ cool efficiently via synchrotron emission. The corresponding RS synchrotron break frequencies evolve as $\nu_{\mathrm{m}, \mathrm{r}}^{\mathrm{syn}} \propto t^{-\frac{73}{48}}$ and $\nu_{\mathrm{c}, \mathrm{r}}^{\mathrm{syn}} \propto t^{\frac{1}{16}}$, while the spectral peak flux density $F_{\max , \mathrm{r}}^{\mathrm{syn}} \propto t^{-\frac{47}{48}}$ (Kobayashi 2000).

\footnotetext{
${ }^{14} \Gamma_{3}=\Gamma / 10^{3}$ is the bulk Lorentz factor at the deceleration time, given by Equation (2), and is not to be confused with $\gamma_{3}$, the fluid Lorentz factor behind the shock.
}

Based on these assessments, the spectral breaks and the maximum flux of SSC emission are given by

$$
\begin{aligned}
h \nu_{\mathrm{m}, \mathrm{r}}^{\mathrm{ssc}} \simeq & 10.1 \mathrm{keV}(1+z)^{-1} \epsilon_{\mathrm{e}_{\mathrm{r}}}^{4} \epsilon_{\mathrm{B}_{\mathrm{r}},-4}^{\frac{1}{2}} n_{-4}^{\frac{3}{4}} \Gamma_{3}^{4} \Delta_{12}^{\frac{45}{16}} E_{\mathrm{K}, 53}^{-\frac{1}{4}} t_{1}^{-\frac{33}{16}} \\
h \nu_{\mathrm{c}, \mathrm{r}}^{\mathrm{ssc}} \simeq & 101.3 \mathrm{TeV}(1+z)^{3} \epsilon_{\mathrm{B}_{\mathrm{r}},-4}^{-\frac{7}{2}} n_{-4}^{-\frac{9}{4}}\left(1+Y_{\mathrm{r}}\right)^{-4} \Delta_{12}^{-\frac{65}{48}} \\
& \times E_{\mathrm{K}, 53}^{-\frac{10}{8}} \frac{53}{\frac{53}{48}} \\
F_{\mathrm{max}, \mathrm{r}}^{\mathrm{ssc}} \simeq & 2.8 \times 10^{-5} \mathrm{mJy} \epsilon_{\mathrm{B}_{\mathrm{r}},-4}^{\frac{1}{2}} \Gamma_{3}^{-1} n_{-4} \\
& \times \Delta_{12}^{\frac{17}{48}} d_{\mathrm{z}, 28}^{-2} E_{\mathrm{K}, 53}^{\frac{3}{2}} t_{1}^{-\frac{41}{48}} .
\end{aligned}
$$

The break energy above the $\mathrm{KN}$ regime is (Wang et al. 2010; Fraija et al. 2019a)

$$
h \nu_{\mathrm{c}, \mathrm{r}}^{\mathrm{KN}} \simeq 16.8 \mathrm{TeV}\left(1+Y_{\mathrm{r}}\right)^{-1} \epsilon_{\mathrm{B}_{\mathrm{r}},-1}^{-1} n_{-4}^{-\frac{3}{4}} E_{\mathrm{KN}, 53}^{-\frac{1}{4}} \Delta_{12}^{-\frac{1}{3}} t_{1}^{\frac{1}{12}} .
$$

Using the Equation (8) and the evolution of the electron Lorentz factors, the SSC light curves for the fast- and the slowcooling regime are given by

$$
F_{\nu, r}^{\mathrm{ssc}} \propto \begin{cases}t^{-\frac{11}{9}} \nu^{\frac{1}{3}}, & \nu<\nu_{\mathrm{c}, \mathrm{r}}^{\mathrm{ssc}}, \\ t^{-\frac{29}{96}} \nu^{-\frac{1}{2}}, & \nu_{\mathrm{c}, \mathrm{r}}^{\mathrm{ssc}}<\nu<\nu_{\mathrm{m}, \mathrm{r}}^{\mathrm{ssc}}, \\ t^{\frac{70-99 p}{96}} \nu^{-\frac{p}{2}}, & \nu_{\mathrm{m}, \mathrm{r}}^{\mathrm{ssc}}<\nu,\end{cases}
$$

and

$$
F_{\nu, r}^{\mathrm{ssc}} \propto \begin{cases}t_{1}^{-\frac{1}{6}} \nu^{\frac{1}{3}}, & \nu<\nu_{\mathrm{m}, \mathrm{r}}^{\mathrm{ssc}}, \\ t^{\frac{17-99 p}{96}} \nu^{-\frac{p-1}{2}}, & \nu_{\mathrm{m}, \mathrm{r}}^{\mathrm{ssc}}<\nu<\nu_{\mathrm{cut}}^{\mathrm{ssc}}, \\ 0, & \nu_{\mathrm{cut}}^{\mathrm{ssc}}<\nu,\end{cases}
$$

respectively, where $\nu_{\mathrm{cut}}^{\mathrm{ssc}}=\nu_{\mathrm{c}, \mathrm{r}}^{\mathrm{ssc}}\left(\frac{t}{t_{\mathrm{x}}}\right)^{-\frac{73}{48}}$. The term $\left(\frac{t}{t_{\mathrm{x}}}\right)^{-\frac{73}{48}}$ is introduced due to the adiabatic expansion of the fluid (Kobayashi 2000). After the peak, the flux above energy $h \nu_{\text {cut }}^{\text {ssc }}$ should disappear. Nonetheless, the angular time delay effect prevents this disappearance (Panaitescu \& Kumar 2000; Kobayashi \& Zhang 2003). During this phase, the evolution of the SSC flux due to high-latitude afterglow emission is described by $F_{\nu, r}^{\text {ssc }} \propto t^{-(\beta+2)}$, where the spectral index ${ }^{15}$ can take the values of $\beta=\frac{1}{2}$ for $\nu_{\mathrm{c}, \mathrm{r}}^{\mathrm{ssc}}<\nu<\nu_{\mathrm{m}, \mathrm{r}}^{\mathrm{ssc}}, \frac{p-1}{2}$ for $\nu_{\mathrm{m}, \mathrm{r}}^{\mathrm{ssc}}<\nu<\nu_{\mathrm{c}, \mathrm{r}}^{\mathrm{ssc}}$ and $\frac{p}{2}$ for $\left\{\nu_{\mathrm{c}, \mathrm{r}}^{\mathrm{ssc}}, \nu_{\mathrm{m}, \mathrm{r}}^{\mathrm{ssc}}\right\}<\nu$. It is worth nothing that, if off-axis flux dominates, the SSC light curves follow the high-latitude afterglow emission (e.g., see Kobayashi et al. 2007).

\subsubsection{The Thin-shell Regime $\left(\Gamma<\Gamma_{c}\right)$}

In the thin-shell regime, the RS becomes mildly relativistic. In this case, the shock crossing time is longer than the duration of the burst $\left(T_{90}<t_{\mathrm{x}}\right)$. As before, we describe the dynamics before and after the shock crossing time, separately.

2.1.2.1. Before the shock crossing time $\left(t<t_{\mathrm{x}}\right)$ Given the evolution of the Lorentz factor of the fluid just behind the RS $\gamma_{3} \propto t^{0}$, the post-shock magnetic field $B^{\prime} \propto t^{0}$ and the minimum and cooling electron Lorentz factors $\gamma_{\mathrm{m}, \mathrm{r}} \propto t^{3}$ and $\gamma_{\mathrm{c}, \mathrm{r}} \propto t^{-1}$, respectively, the corresponding synchrotron break

\footnotetext{
15 Hereafter, we use, in general, the notation $F_{\nu} \propto t^{-\alpha} \nu^{-\beta}$ for the closure relations.
} 
frequencies at the RS region evolve as $\nu_{\mathrm{m}, \mathrm{r}}^{\mathrm{syn}} \propto t^{6}$ and $\nu_{\mathrm{c}, \mathrm{r}}^{\mathrm{syn}} \propto t^{-2}$ and the maximum flux in terms of the spectrum as $F_{\text {max,r }}^{\mathrm{syn}} \propto t^{\frac{3}{2}}$ (Kobayashi 2000).

For this time interval, the spectral breaks and the maximum flux of SSC emission are given by

$$
\begin{aligned}
h \nu_{\mathrm{m}, \mathrm{r}}^{\mathrm{ssc}} \simeq & 1.8 \times 10^{2} \mathrm{eV}(1+z)^{-1} \epsilon_{\mathrm{e}_{\mathrm{r}},-1}^{4} \epsilon_{\mathrm{B}_{\mathrm{r}},-1}^{\frac{1}{2}} \\
& \times n_{-1}^{\frac{9}{2}} \Gamma_{2}^{34} E_{\mathrm{K}, 51}^{-4} t_{2}^{12} \\
h \nu_{\mathrm{c}, \mathrm{r}}^{\mathrm{ssc}} \simeq & 0.3 \mathrm{GeV}(1+z)^{3}\left(1+Y_{\mathrm{r}}\right)^{-4} \epsilon_{\mathrm{B}_{\mathrm{r}},-1}^{-\frac{7}{2}} n_{-1}^{-\frac{7}{2}} \Gamma_{2}^{-10} t_{2}^{-4}, \\
F_{\text {max }, \mathrm{r}}^{\mathrm{ssc}} \simeq & 1.4 \times 10^{-4} \mathrm{mJy} \epsilon_{\mathrm{B}_{\mathrm{r}},-1}^{\frac{1}{2}} n_{-1}^{2} \Gamma_{2}^{7} d_{\mathrm{z}, 28}^{-2} E_{\mathrm{K}, 51}^{\frac{1}{2}} t_{2}^{\frac{5}{2}},
\end{aligned}
$$

where the bulk Lorentz factor is less than the critical one $\left(\Gamma<\Gamma_{c} \simeq 161.9 E_{\mathrm{K}, 51}^{\frac{1}{8}} n_{-1}^{-\frac{1}{8}} T_{90}^{-\frac{3}{8}}\right.$ for $\left.T_{90}=50 \mathrm{~s}\right)$.

The break energy above the $\mathrm{KN}$ regime is

$$
h \nu_{\mathrm{c}, \mathrm{r}}^{\mathrm{KN}} \simeq 111.6 \mathrm{GeV}\left(1+Y_{\mathrm{r}}\right)^{-1} \epsilon_{\mathrm{B}_{\mathrm{r}},-1}^{-1} n_{-1}^{-1} \Gamma_{2}^{-2} t_{2}^{-1} .
$$

In analogy to the description of the SSC light curves for the thick-shell regime, the SSC light curves before the shock crossing time for the fast- and the slow-cooling regime are

$$
F_{\nu, r}^{\mathrm{ssc}} \propto \begin{cases}t^{\frac{23}{6}} \nu^{\frac{1}{3}}, & \nu<\nu_{\mathrm{c}, \mathrm{r}}^{\mathrm{ssc}}, \\ t^{\frac{1}{2}} \nu^{-\frac{1}{2}}, & \nu_{\mathrm{c}, \mathrm{r}}^{\mathrm{ssc}}<\nu<\nu_{\mathrm{m}, \mathrm{r}}^{\mathrm{ssc}}, \\ t^{\frac{12 p-11}{2}} \nu^{-\frac{p}{2}}, & \nu_{\mathrm{m}, \mathrm{r}}^{\mathrm{ssc}}<\nu\end{cases}
$$

and

$$
F_{\nu, r}^{\mathrm{ssc}} \propto \begin{cases}t^{-\frac{3}{2}} \nu^{\frac{1}{3}}, & \nu<\nu_{\mathrm{c}, \mathrm{r}}^{\mathrm{ssc}}, \\ t^{\frac{12 p-7}{2}} \nu^{-\frac{p-1}{2}}, & \nu_{\mathrm{m}, \mathrm{r}}^{\mathrm{ssc}}<\nu<\nu_{\mathrm{c}, \mathrm{r}}^{\mathrm{ssc}}, \\ t^{\frac{12 p-11}{2}} \nu^{-\frac{p}{2}}, & \nu_{\mathrm{c}, \mathrm{r}}^{\mathrm{ssc}}<\nu .\end{cases}
$$

2.1.2.2. After the shock crossing time $\left(t>t_{\mathrm{x}}\right)$ Given the evolution of the post-shock magnetic field $B^{\prime} \propto t^{-\frac{4}{7}}$ and the minimum and cooling electron Lorentz factors $\gamma_{\mathrm{m}, \mathrm{r}} \propto t^{-\frac{2}{7}}$ and $\gamma_{\mathrm{c}, \mathrm{r}} \propto t^{\frac{19}{35}}$, respectively, the corresponding synchrotron break frequencies at the $\mathrm{RS}$ region evolve as $\nu_{\mathrm{m}, \mathrm{r}}^{\mathrm{syn}} \propto t^{-\frac{54}{35}}$ and $\nu_{\mathrm{c}, \mathrm{r}}^{\mathrm{syn}} \propto t^{\frac{4}{35}}$ and the maximum flux in terms of the spectrum as $F_{\max , \mathrm{r}}^{\text {syn }} \propto t^{-\frac{34}{35}}$ (Kobayashi 2000).

Following the same previous process, the spectral breaks and the maximum flux of SSC emission are given by

$$
\begin{aligned}
h \nu_{\mathrm{m}, \mathrm{r}}^{\mathrm{ssc}} \simeq & 1.3 \times 10^{-2} \mathrm{eV}(1+z)^{-1} \epsilon_{\mathrm{e}_{\mathrm{r}},-1}^{4} \epsilon_{\mathrm{B}_{\mathrm{r}},-1}^{\frac{1}{2}} n_{-1}^{-\frac{43}{20}} \Gamma_{2}^{-\frac{382}{105}} \\
& \times E_{\mathrm{K}, 51}^{\frac{74}{105}} t_{2}^{-\frac{74}{35}} \\
h \nu_{\mathrm{c}, \mathrm{r}}^{\mathrm{ssc}} \simeq & 10.2 \mathrm{TeV}(1+z)^{3}\left(1+Y_{\mathrm{r}}\right)^{-4} \epsilon_{\mathrm{B}_{\mathrm{r}},-1}^{-\frac{7}{2}} n_{-1}^{-\frac{53}{30}} \Gamma_{2}^{\frac{58}{15}} \\
& \times E_{\mathrm{K}, 51}^{-\frac{26}{15}} t_{2}^{\frac{6}{5}} \\
F_{\text {max, }}^{\mathrm{ssc}} \simeq & 7.2 \times 10^{-5} \mathrm{mJy} \epsilon_{\mathrm{B}_{\mathrm{r}},-1}^{\frac{1}{2}} n_{-1}^{\frac{191}{210}} \Gamma_{2}^{-\frac{181}{105}} \\
& \times d_{\mathrm{z}, 28}^{-2} E_{\mathrm{K}, 51}^{\frac{167}{105}} t_{2}^{-\frac{27}{35}} .
\end{aligned}
$$

The break energy above the $\mathrm{KN}$ regime is given by Wang et al. (2010) and Fraija et al. (2019a):

$$
h \nu_{\mathrm{c}, \mathrm{r}}^{\mathrm{KN}} \simeq 1.9 \mathrm{TeV}\left(1+Y_{\mathrm{r}}\right)^{-1} \epsilon_{\mathrm{B}_{\mathrm{r}},-1}^{-1} n_{-1}^{-\frac{11}{15}} \Gamma_{2}^{\frac{2}{15}} E_{\mathrm{K}, 51}^{-\frac{4}{5}} t_{2}^{-\frac{1}{5}} .
$$

In analogy to the description of the SSC light curves for the thick-shell regime, the SSC light curves after the shock crossing time for the fast- and the slow-cooling regime are

$$
F_{\nu, r}^{\mathrm{ssc}} \propto \begin{cases}t^{-\frac{41}{35}} \nu^{\frac{1}{3}}, & \nu<\nu_{\mathrm{c}, \mathrm{r}}^{\mathrm{ssc}}, \\ t^{-\frac{6}{35}} \nu^{-\frac{1}{2}}, & \nu_{\mathrm{c}, \mathrm{r}}^{\mathrm{ssc}}<\nu<\nu_{\mathrm{m}, \mathrm{r}}^{\mathrm{ssc}}, \\ t^{\frac{31-37 p}{35}} \nu^{-\frac{p}{2}}, & \nu_{\mathrm{m}, \mathrm{r}}^{\mathrm{ssc}}<\nu,\end{cases}
$$

and

$$
F_{\nu, r}^{\mathrm{ssc}} \propto \begin{cases}t^{-\frac{1}{15}} \nu^{\frac{1}{3}}, & \nu<\nu_{\mathrm{m}, \mathrm{r}}^{\mathrm{ssc}}, \\ t^{\frac{10-37 p}{35}} \nu^{-\frac{p-1}{2}}, & \nu_{\mathrm{m}, \mathrm{r}}^{\mathrm{ssc}}<\nu<\nu_{\mathrm{cut}}^{\mathrm{ssc}}, \\ 0, & \nu_{\mathrm{cut}}^{\mathrm{ssc}}<\nu .\end{cases}
$$

The SSC emission from the RS region could decay faster due to the angular time delay effect, and the corresponding lightcurve evolution is the same as that discussed at the end of Section 2.1.1.

\subsection{Synchrotron Light Curves from FS}

The dynamics of the FS for a relativistic outflow expanding into a uniform-density medium is explained in Sari et al. (1998). Using the evolution of synchrotron energy breaks $\left(\nu_{\mathrm{m}, \mathrm{f}}^{\mathrm{syn}} \propto t^{-\frac{3}{2}}\right.$ and $\left.\nu_{\mathrm{c}, \mathrm{f}}^{\text {syn }} \propto t^{-\frac{1}{2}}\right)$ and the maximum flux $\left(F_{\text {max,f }}^{\text {syn }} \propto t^{0}\right)$, the observed flux in the fast-cooling regime is proportional to $\propto t^{-\frac{1}{4}} \nu^{-\frac{1}{2}}$ for $\nu<\nu_{\mathrm{m}, \mathrm{f}}^{\mathrm{syn}}$ and $\propto t^{-\frac{3 p-2}{4}} \nu^{-\frac{p}{2}}$ for $\nu_{\mathrm{m}, \mathrm{f}}^{\mathrm{syn}}<\nu$. Hereafter, the subindex "f $\mathrm{f}$ " refers to the derived quantities in the FS. In the slow-cooling regime, the observed flux is proportional to $\propto t^{-\frac{3 p-3}{4}} \nu^{-\frac{p-1}{2}}$ for $\nu<\nu_{\mathrm{c}, \mathrm{f}}^{\mathrm{syn}}$ and $\propto t^{-\frac{3 p-2}{4}} \nu^{-\frac{p}{2}}$ for $\nu_{\mathrm{c}, \mathrm{f}}^{\text {syn }}<\nu$, where the proportionality factors are explicitly written in, e.g., Fraija et al. (2016b).

\subsection{The GeV Flares and the Break in the Long-lasting Emission}

Figure 1 shows the theoretically predicted SSC and synchrotron light curves from RS and FS evolving in the fastand slow-cooling regimes, respectively. The predicted SSC light curves are presented in the thick- (left column) and thin(right column) shell regimes for a uniform-density medium. The light curves in Figure 1 from top to bottom display the $\mathrm{SSC}$ flux for $\nu_{\mathrm{c}, \mathrm{r}}^{\mathrm{ssc}}<\nu<\nu_{\mathrm{m}, \mathrm{r}}^{\mathrm{ssc}}$ and $\nu_{\mathrm{m}, \mathrm{r}}^{\mathrm{ssc}}<\nu$ (in the fast-cooling regime) followed by the SSC flux for $\nu_{\mathrm{m}, \mathrm{r}}^{\mathrm{ssc}}<\nu<\nu_{\mathrm{c}, \mathrm{r}}^{\mathrm{ssc}}$ and $\nu_{\mathrm{c}, \mathrm{r}}^{\mathrm{ssc}}<\nu$ (in the slow-cooling regime). We do not discuss the effects at the self-absorption regime, because its contribution is typically significant only at low energies. (e.g., see Panaitescu et al. 2014). Similarly, we do not analyze the SSC light curves for $\nu<\min \left\{\nu_{\mathrm{m}, \mathrm{r}}^{\mathrm{ssc}}, \nu_{\mathrm{c}, \mathrm{r}}^{\mathrm{ssc}}\right\}$, because they are relevant at optical and radio bands (Kobayashi \& Zhang 2003) and not at energies around $100 \mathrm{MeV}$. Radiation from high latitudes received after the shock crossing time may prevent the abrupt disappearance of the RS emission (Kobayashi 2000). Transitions from fast- to slow-cooling regimes and from wind to uniform-density media have not been considered in these light curves.

When the observed light curves consist of a superposition of SSC from the RS and the synchrotron emission from the FS, as described here, we would naturally expect that the traditional closure relations between the light curve evolution and spectral index would not be satisfied. Only when the SSC emission is 

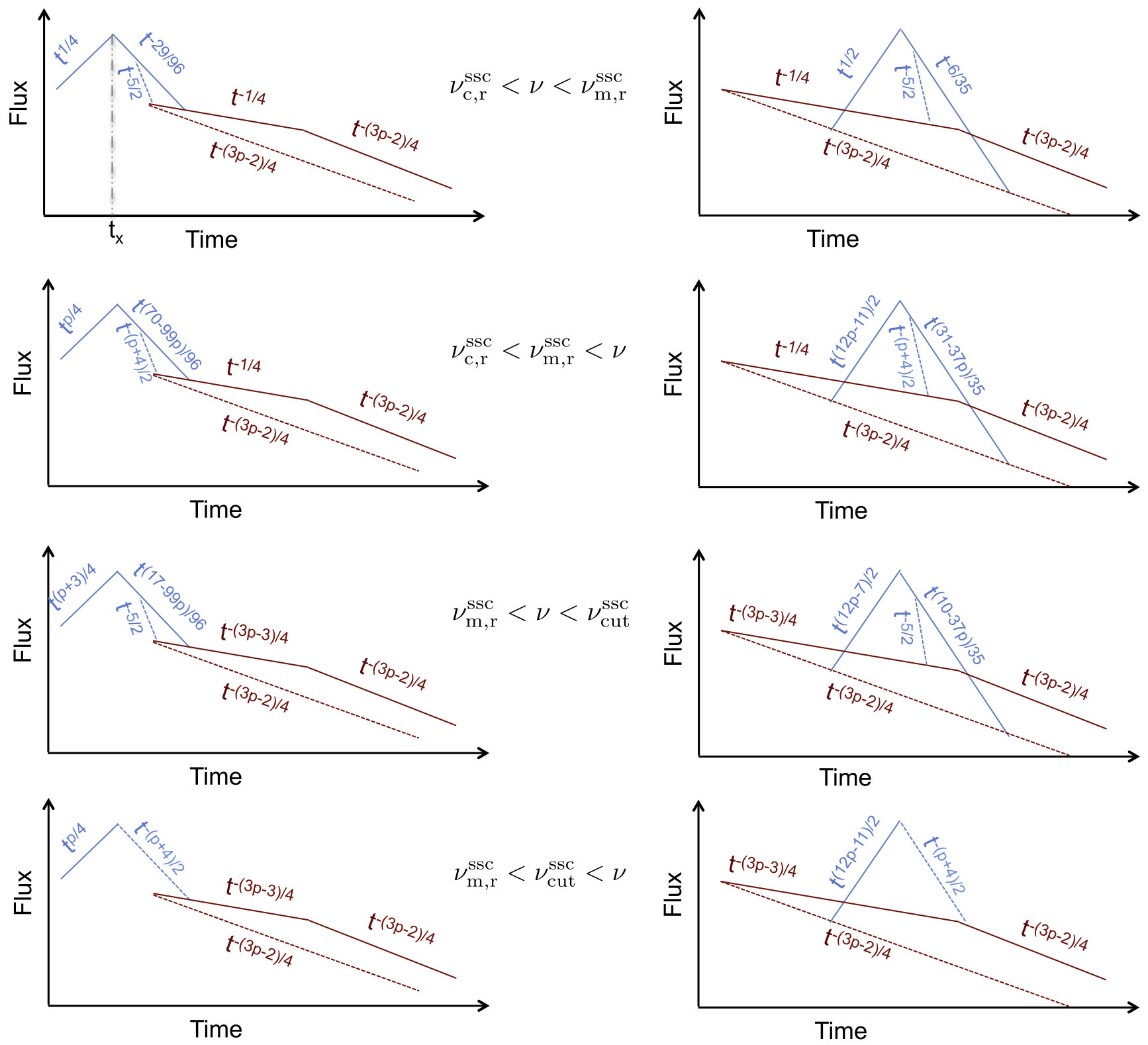

Figure 1. Theoretically predicted SSC light curves from RS (blue lines) together with the synchrotron light curves from FSs (red lines) evolving in the fast- and slowcooling regimes. Predicted SSC light curves are presented in the thick- (left column) and thin- (right column) shell regimes for a uniform-density medium. Light curves from top to bottom display the SSC flux for $\nu_{\mathrm{c}, \mathrm{r}}^{\mathrm{ssc}}<\nu<\nu_{\mathrm{m}, \mathrm{r}}^{\mathrm{ssc}}$ and $\epsilon_{\mathrm{m}, \mathrm{r}}^{\mathrm{ssc}}<\nu$ (in the fast-cooling regime) followed by the SSC flux for $\nu_{\mathrm{m}, \mathrm{r}}^{\mathrm{ssc}}<\nu<\nu_{\mathrm{cut}}^{\mathrm{ssc}}$ and $\nu_{\text {cut }}^{\text {ssc }}<\nu_{\gamma}$ (in the slow-cooling regime). Dot-dot-dashed line in gray refers to shock crossing time $\left(t_{\mathrm{x}}\right)$. Blue dashed lines indicate an alternative PL evolution of the SSC model (see the SSC light curves in Section 2.1). Breaks exhibited in the solid lines (synchrotron light curves) correspond to the transitions between $\nu<\nu_{\mathrm{c}, \mathrm{f}}^{\mathrm{syn}}$ and $\nu_{\mathrm{c}, \mathrm{f}}^{\mathrm{syn}}<\nu$. Red dashed lines indicate that, initially, synchrotron light curves lie in the range $\nu_{\mathrm{c}, \mathrm{f}}^{\mathrm{syn}}<\nu$, thus no breaks are expected.

suppressed or has decreased below the FS synchrotron emission would we expect the closure relation to be satisfied. It is worth noting that, depending on the parameter values, synchrotron radiation could dominate over SSC emission. The SSC light curves from RS indicate that rise $\left(\alpha_{\text {ris }}\right)$ and decay $\left(\alpha_{\mathrm{dec}}\right)$ indices could be expected between $-\frac{p+3}{4} \lesssim \alpha_{\text {ris }} \lesssim-\frac{1}{4}$ and $\frac{29}{96} \lesssim \alpha_{\text {dec }} \lesssim \frac{p+4}{2}$ for a thick-shell regime, and between $-\frac{12 p-7}{2} \lesssim \alpha_{\text {ris }} \lesssim-\frac{1}{2}$ and $\frac{6}{35} \lesssim \alpha_{\text {dec }} \lesssim \frac{p+4}{2}$ for a thin-shell regime, respectively. For instance, with a typical value of the spectral index of $p=2.4$, the temporal rise and decay index for $\nu_{\mathrm{m}, \mathrm{r}}^{\mathrm{ssc}}<\nu<\nu_{\mathrm{c}, \mathrm{r}}^{\mathrm{ssc}}$ is $\alpha_{\mathrm{ris}} \simeq-1.35$ and $\alpha_{\mathrm{dec}}=2.50$ for the thickshell regime, and $\alpha_{\text {ris }} \simeq-10.90$ and $\alpha_{\mathrm{dec}}=2.50$ for the thinshell regime, respectively.
The FS synchrotron light curves in the LAT band for a uniform-density medium show different behaviors associated with transitions between distinct PL segments (Figure 1). The breaks predicted in the synchrotron light curves correspond to the transitions from $\nu<\nu_{\mathrm{m}, \mathrm{f}}^{\mathrm{syn}}$ to $\nu_{\mathrm{m}, \mathrm{f}}^{\mathrm{syn}}<\nu$ in the fast-cooling regime, and from $\nu<\nu_{\mathrm{c}, \mathrm{f}}^{\mathrm{syn}}$ to $\nu_{\mathrm{c}, \mathrm{f}}^{\mathrm{syn}}<\nu$ in the slow-cooling regime. The synchrotron spectral breaks evolve as $\nu_{\mathrm{m}, \mathrm{f}}^{\mathrm{syn}} \propto t^{-\frac{3}{2}}$ and $\nu_{\mathrm{c}, \mathrm{f}}^{\mathrm{syn}} \propto t^{-\frac{1}{2}}$, such that transitions between these PL segments in the fast- and the slow-cooling regime are expected to be associated with changes in the temporal indexes and a steepening in the light curve. Considering an electron energy index of $p=2.4$, the temporal index, in general, varies from 0.25 to 1.30 and from 1.05 to 1.30 for the fast- and the slowcooling regimes, respectively (Sari et al. 1998). 

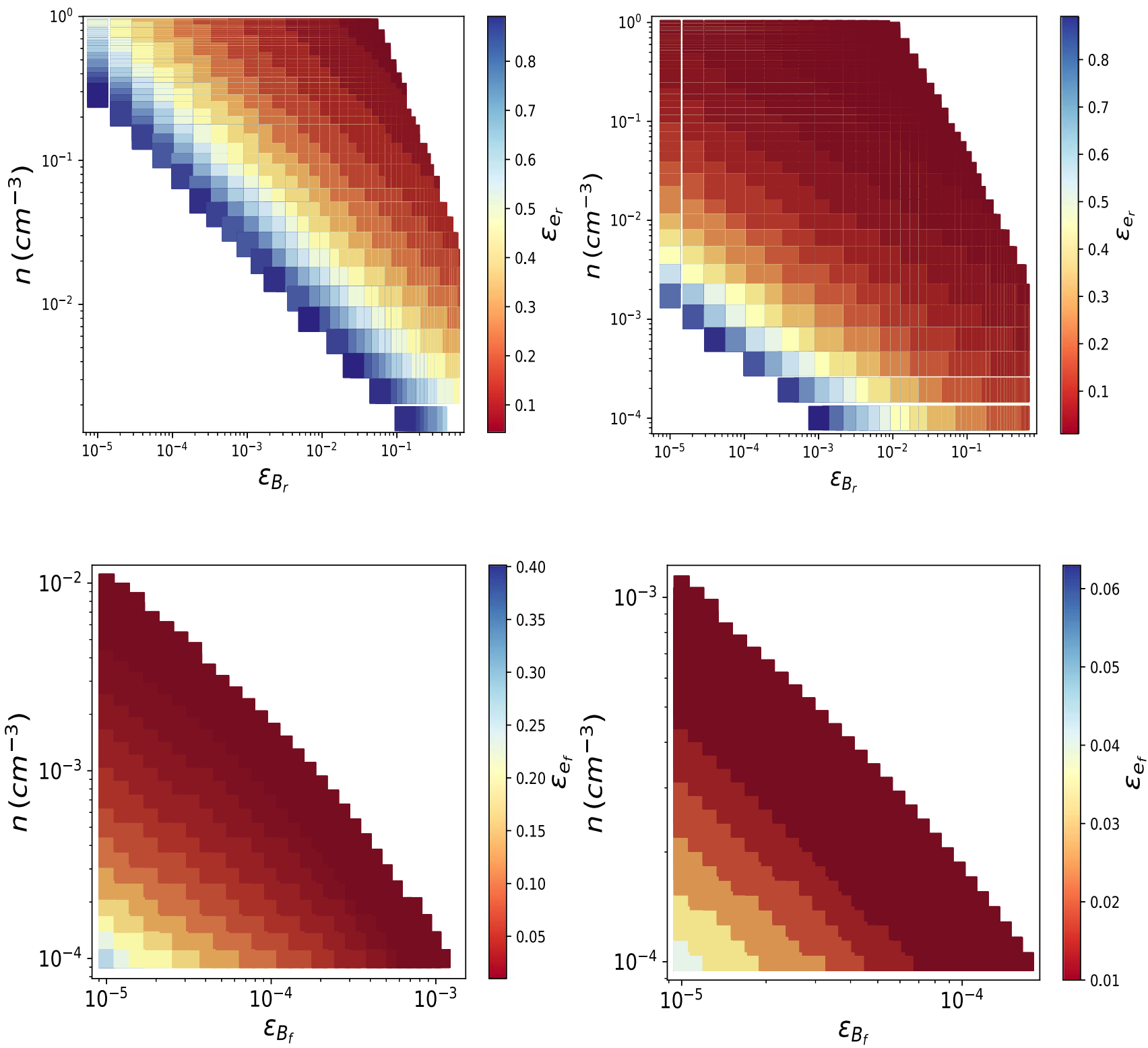

Figure 2. Upper panels: range of microphysical parameters and density for which the SSC emission is above $5 \times 10^{-5} \mathrm{mJy}$ for $E=10^{52} \mathrm{erg}\left(\right.$ left) and $E=10^{54} \mathrm{erg}$ (right). Value of the threshold flux was estimated considering the sensitivity of Fermi LAT reported by Piron (2016) and de Angelis et al. (2017). Lower panels: range of microphysical parameters and density for which the FS synchrotron emission exhibits a break between 100 and $500 \mathrm{~s}$ due to the passage of $\nu_{\mathrm{c}, \mathrm{f}}^{\mathrm{syn}}$ for $E=10^{52}$ erg (left) and $E=10^{54} \mathrm{erg}$ (right) for $\mathrm{p}=2.2$.

We argue that a LAT-detected burst that exhibits a GeV flare and a break in the long-lasting emission can be interpreted in terms of external shock emission: the GeV flare as SSC emission from the RS and the break in the long-lasting emission as the transition between PL segments of synchrotron radiation from the FS. A bright peak from the RS is expected at the RS shock crossing time, $t_{\mathrm{x}}$. In the thick-shell regime, $t_{\mathrm{x}}<T_{90}$, resulting in the RS SSC peak occurring prior to the onset of the FS emission. Conversely, in the thin-shell regime where $t_{\mathrm{x}} \gtrsim T_{90}$, the RS SSC peak overlaps with FS emission.

As an illustration, we show the parameter space of the microphysical parameters and the density of the circumburst medium for which (i) the RS SSC emission is in the thick-shell regime at $10 \mathrm{~s}$, with a flux density $>5 \times 10^{-5} \mathrm{mJy}$ at $100 \mathrm{MeV}$ (Figure 2, upper panels), and (ii) the FS cooling frequency, $\nu_{\mathrm{c}, \mathrm{f}}^{\mathrm{syn}}$ crosses $100 \mathrm{MeV}$ between 100 and $500 \mathrm{~s}$ after the onset of the burst (Figure 2, lower panels), resulting in a break in the $100 \mathrm{MeV}$ light curve. We explore two different values of the isotropic-equivalent kinetic energy, $E_{\mathrm{K}}=10^{52} \mathrm{erg}$ (left column) and $E_{\mathrm{K}}=10^{54} \mathrm{erg}$ (right column). The time interval of $100-500 \mathrm{~s}$ is chosen to explore the breaks observed in some bursts (e.g., GRB 160509A; Ajello et al. 2019). The value of the threshold flux was estimated considering the sensitivity of Fermi-LAT reported by Piron (2016) and de Angelis et al. (2017). It is worth noting that the parameter $\epsilon_{\mathrm{e}_{\mathrm{f}}}$ is quite strongly constrained from radio peaks in GRB afterglows (e.g., see Beniamini \& van der Horst 2017).

In the model with $E_{K}=10^{52} \mathrm{erg}$, there are no values of the physical parameters for which both a GeV flare RS SSC emission and a break in the FS synchrotron radiation due to the passage of $\nu_{\mathrm{c}, \mathrm{f}}^{\mathrm{syn}}$ are simultaneously observed, as long as $R_{\mathrm{e}} \equiv \frac{\epsilon_{\mathrm{ef}}}{\epsilon_{\mathrm{er}}} \approx 1$ and $R_{\mathrm{B}} \equiv\left(\frac{\epsilon_{\mathrm{Br}}}{\epsilon_{\mathrm{Bf}}}\right)^{\frac{1}{2}} \approx 1$. In the model with $E_{\mathrm{K}}=10^{54} \mathrm{erg}$, the two phenomena can be observed in the 
same burst, provided the density is low $\left(n \approx 10^{-3} \mathrm{~cm}^{-3}\right)$ and the RS region is highly magnetized $\left(R_{\mathrm{B}} \gg 1\right)$. Indeed, such large magnetizations are expected in magnetically dominated models for the GRB emission (e.g., Giannios 2008; Zhang \& Yan 2011; McKinney \& Uzdensky 2012; Beniamini \& Piran 2014; Sironi 2015; Beniamini \& Granot 2016; Bégué et al. 2017; Beniamini \& Giannios 2017; Beniamini et al. 2018). Similarly, it can be inferred that the GeV flare in the LAT light curves is unlikely to be in a weak (e.g., lowluminosity) GRB, even in a low-density environment. Moderately high values of magnetization also have been inferred from several multiwavelength RS studies, GRB 130427A $\left(\mathcal{R}_{\mathrm{B}} \approx 1-5\right.$; Laskar et al. 2013; Perley et al. 2014), GRB 160509A $\quad\left(\mathcal{R}_{\mathrm{B}} \approx 3\right.$; Laskar et al. 2016), GRB $160625 B\left(\mathcal{R}_{B} \approx 1-10\right.$; Alexander et al. 2017), although see also Laskar et al. (2019) for a system with $\mathcal{R}_{\mathrm{B}}=0.6 \pm 0.1$ detected by Fermi-LAT. We thus infer that the presence of simultaneous RS SSC emission and a break in the FS MeV light curve suggest a system with $R_{\mathrm{e}} \gtrsim 1$ and/or a combination of high $E_{\mathrm{K}}$, a low-density environment, and strong relative magnetization $R_{\mathrm{B}} \gtrsim 1$ between the RS and FS.

We now apply these principles to investigate the LAT light curve of GRB 160509A. Later on, in Section 4, we provide high-energy light curve predictions in the thin-shell regime, using the example of GRB 180418A.

\section{GRB 160509A: The Thick-shell Case}

At 08:59:04.36 UTC on 2016 May 09, both instruments on board the Fermi satellite, namely the GBM (Gamma Burst Monitor) and the LAT, triggered and located GRB 160509A (Roberts et al. 2016; Longo et al. 2016). The burst was located with coordinates R.A. $=310.1$ and Dec $=76.0($ J2000) with an error radius of $0.50^{\circ}(90 \%$ containment, the systematic error only). The LAT instrument detected a very energetic photon with an energy of $52 \mathrm{GeV}$, at $\approx 77 \mathrm{~s}$ after the trigger. The GBM light curve in the 50-300 keV energy range exhibited multiple peaks, with a duration of $T_{90}=371 \mathrm{~s}$. The Gemini North telescope observed this burst at 13:15 UT on 10 May 2016, obtaining optical spectroscopy and near-IR imaging with the GMOS-N and NIRI instruments, respectively. A single and well-defined emission line ([OII] $3727 \AA$ ) in the spectroscopic analysis indicated a redshift of $z=1.17$ (Tanvir et al. 2016). In the radio bands, this burst was detected with the VLA (Very Large Array) at frequencies spanning between 1.3 and $37 \mathrm{GHz}$, beginning 0.36 days after the trigger time (Laskar et al. 2016).

\subsection{Fermi-LAT Observations}

The Fermi-LAT light curve of GRB $160509 \mathrm{~A}^{16}$ exhibits two components: a GeV flare with a duration of $\sim 20$ s and a longlasting emission that begins $\sim 40 \mathrm{~s}$ after the LAT trigger and extends for more than $\sim 10^{3}$ s (Figure 3 ; red points). We fit the light curve with a series of broken PLs (next two sections) and discuss each component separately. The best-fit values of the $\mathrm{GeV}$ flare and the long-lasting emission are reported in Table 1. It is worth noting that, in Ajello et al. (2019), the function defined by Willingale et al. (2007) was used to explore the existence of the plateau phase and to model it simultaneously with the prompt emission component. The best-fit

\footnotetext{
16 LAT data points are taken from https://www-glast.stanford.edu/pub_data/ $953 /$. The energy range of $0.1-100 \mathrm{GeV}$ integrated over the spectrum is used to convert from erg $\mathrm{cm}^{-2} \mathrm{~s}^{-1}$ to $\mu \mathrm{Jy}$ at $100 \mathrm{MeV}$.
}

values reported for the PL indices before and after the break time were $0.9 \pm 0.3$ and $1.3 \pm 0.3$, respectively (see Table 5 in Ajello et al. 2019). Thus, regardless of the function used, the presence of two components is visible.

\subsubsection{The GeV Flare}

To describe the GeV flare, two PL functions were used: $F_{\mathrm{L}, \mathrm{pk}} \propto\left(t-t_{0}\right)^{-\alpha_{\mathrm{L}, \mathrm{ris}}}$ for $t<t_{\mathrm{pk}}$ and $\propto t^{-\alpha_{\mathrm{L}, \mathrm{dec}}}$ for $t_{\mathrm{pk}}<t$. The term $t_{0}$ is the starting time of the GeV flare (Vestrand et al. 2006; Kobayashi \& Zhang 2007), $t_{\mathrm{pk}} \approx 20 \mathrm{~s}$ is the time for which the flux reaches the maximum value and begins decreasing, and $\alpha_{\mathrm{L} \text {,ris }}$ and $\alpha_{\mathrm{L} \text {,dec }}$ are the temporal rise and decay indices, respectively. Using linear least squares (Lai et al.

1978) fitting implemented in ROOT, which is a modular, publicly available scientific software package (Brun \& Rademakers 1997), we found that the best-fit values are $t_{0}=8.4 \pm 0.5 \mathrm{~s}, \quad \alpha_{\mathrm{L}, \mathrm{ris}}=-(1.25 \pm 0.21) \quad$ and $\alpha_{\mathrm{L}, \mathrm{dec}}=3.14 \pm 0.65$.

Maxham et al. (2011) investigated an FS origin for the GeV light curves of four GRBs (080916C, 090510, 090902B, and 090926A). They demonstrated that at $\mathrm{t} \gg T_{90}$, FS emission could explain the GeV light curve. However, the FS synchrotron contribution usually underpredicts the GeV light curve while the central engine is active (i.e., at $t \lesssim T_{90}$ ), due to the expectation of ongoing energy accumulation in the FS over this period. We note that the GeV flare in GRB 160509A occurs at $t \lesssim T_{90}$. We posit that a possible mechanism to reconcile the discrepancy noted by Maxham et al. (2011) may lie in RS SSC emission.

Comparing the best-fit value $\alpha_{\mathrm{L}, \mathrm{ris}}=-(1.25 \pm 0.21)$ with the temporal indexes of the SSC light curves for $t<t_{\mathrm{x}}$ (Equation (16)), we notice that it is consistent with SSC model in the range $\nu_{\mathrm{c}, \mathrm{r}}^{\mathrm{ssc}}<\nu<\nu_{\mathrm{m}, \mathrm{r}}^{\mathrm{ssc}}$ for $p=2.24 \pm 0.40$. Other PL segments in the SSC light curves would produce atypical values of $p$. Given the spectral index $p=2.24 \pm 0.40$ when the SSC emission evolves in the range $\nu_{\mathrm{c}, \mathrm{r}}^{\mathrm{ssc}}<\nu<\nu_{\mathrm{m}, \mathrm{r}}^{\mathrm{ssc}}$, after the shock crossing time, the flux decays as $2.13 \pm 0.42$ (Equation (8)), and finally $2+\beta=2.62 \pm 0.20$ due to highlatitude emission. Other PL segments of the SSC light curve cannot reproduce the decaying phase. Therefore, the best-fit parameters of the rise and decay PL indexes indicate that the GeV flare is consistent with an RS evolving in the uniformdensity medium and the thick-shell regime. The best-fit values of each component are reported in Table 1.

\subsubsection{The Long-lasting Emission}

The long-lasting emission component is described with a broken PL function of the form

$$
F_{\mathrm{L}, \text { ee }}(t) \propto \begin{cases}t^{-\alpha_{\mathrm{L}, 1}} & t<t_{\mathrm{L}, \mathrm{br}} \\ t^{-\alpha_{\mathrm{L}, 2}} & t_{\mathrm{L}, \mathrm{br}}<t .\end{cases}
$$

The parameters $\alpha_{\mathrm{L}, 1}$ and $\alpha_{\mathrm{L}, 2}$ are the temporal indices before and after the time break $t_{\mathrm{L}, \mathrm{br}}$. We fit this model to the LAT light curve at $43-1081$ using the chi-squared method (Brun \& Rademakers 1997). Our best-fit values are $t_{\mathrm{L}, \mathrm{br}}=316.2 \pm 61.2 \mathrm{~s}, \quad \alpha_{\mathrm{L}, 1}=0.87 \pm 0.18 \quad$ and $\alpha_{\mathrm{L}, 2}=1.26 \pm 0.28$, which are consistent with those reported in the second LAT GRB catalog (see Figure 27 in Ajello et al. 2019).

On the other hand, Tam et al. (2017) analyzed the LAT spectrum of the long-lasting emission. Using a PL function 


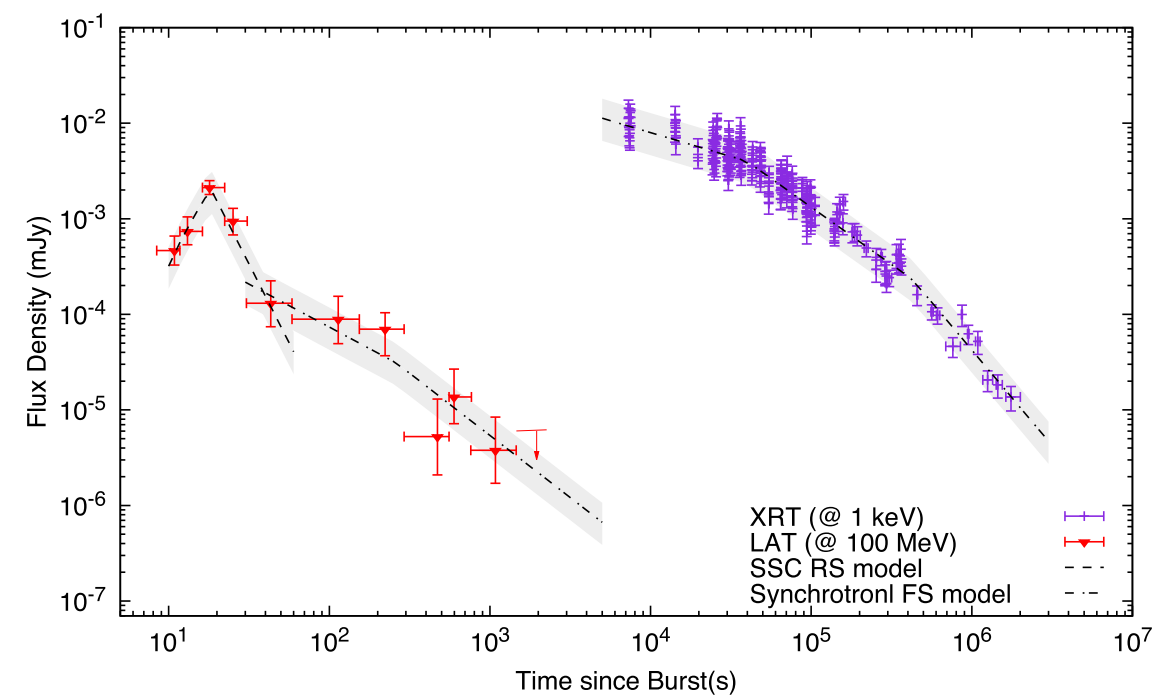

Figure 3. Multiwavelength light curves of GRB 160509A, with the synchrotron FS and SSC RS models. Synchrotron FS model was used to describe the long-lived LAT (dashed-dotted line) and X-ray (dotted line) observations, and the SSC RS model was used to interpret the GeV flare (dashed line). Hatched area shows the uncertainty of the best-fit parameters obtained with the MCMC (see Table 3).

Table 1

Best-fit Values Found from the LAT Light Curve of GRB 160509A

\begin{tabular}{lccc}
\hline \hline LAT & Parameter & Best-fit value & $\chi^{2} / \mathrm{ndf}$ \\
\hline GeV flare & & & \\
& $\alpha_{\mathrm{L}, \mathrm{ris}}$ & $1.25 \pm 0.21$ & 1.22 \\
& $\alpha_{\mathrm{L}, \mathrm{dec}}$ & $3.14 \pm 0.65$ & \\
& $t_{0}(\mathrm{~s})$ & $8.4 \pm 0.5$ & \\
\hline Extended emission & & & \\
& $\alpha_{L, 1}$ & $0.87 \pm 0.18$ & 1.27 \\
& $\alpha_{L, 2}$ & $1.26 \pm 0.21$ & \\
& $t_{\mathrm{L}, \mathrm{br}}(\mathrm{s})$ & $316.2 \pm 61.2$ & \\
\hline
\end{tabular}

$\frac{d N}{d E} \propto E^{-\Gamma_{\mathrm{L}}}$ Tam et al. (2017) reported two distinct spectral indices: $\Gamma_{\mathrm{L}, 1}=\beta_{\mathrm{L}, 1}+1=1.42 \pm 0.12$ for $40 \lesssim t \lesssim 300 \mathrm{~s}$ and $\Gamma_{\mathrm{L}, 2}=2.2 \pm 0.3$ for $300 \lesssim t \lesssim 10^{3} \mathrm{~s}$. Therefore, this component of the Fermi LAT observations can be described by $F_{\nu, f}^{\text {syn }} \propto t^{-0.87 \pm 0.18} \nu^{-0.42 \pm 0.12} \quad$ before $\lesssim 300 \mathrm{~s} \quad$ and $F_{\nu, f}^{\text {syn }} \propto t^{-1.26 \pm 0.28} \nu^{-1.2 \pm 0.3}$ after $\gtrsim 300 \mathrm{~s}$. In the synchrotron framework (Section 2.2), the passage of the synchrotron cooling frequency is expected to result in the steepening of the light curve by $\delta \alpha \approx 0.25$ and a change in the spectral index by $|\delta \beta| \approx 0.5$, both of which are consistent with the observed evolution in the LAT light curve and spectrum across $t_{\mathrm{L}, \mathrm{br}}$. Furthermore, the sign of $\delta \beta$ across the break is a diagnostic of the density profile. The steepening seen here is indicative of a uniform-density environment. This is also consistent with the inference of Laskar et al. (2016), who infer a uniform-density environment based on modeling the RS emission at radio wavelengths for this burst. Taking $(2-p) / 4=1.26 \pm 0.28$ at $\gtrsim t_{\mathrm{L}, \mathrm{br}}$ (where the data are less affected by potential contamination from the earlier GeV flare), we find $p=2.35 \pm 0.37$. This yields $\beta_{\mathrm{L}, 2}=1.2 \pm 0.2$, consistent with the observed value. Before the break, we expect a decay rate of $\alpha_{\mathrm{L}, 1}=3(p-1) / 4=1.01 \pm 0.28$ and spectral slope of $\beta_{\mathrm{L}, 1}=(p-1) / 2=0.68 \pm 0.19$, consistent with observations. Therefore, the Fermi-LAT observations are consistent with an FS emission in a uniform-density environment, with the spectral ordering $\nu_{m}<\nu_{\mathrm{LAT}}<\nu_{\mathrm{c}}$ at $\lesssim 316 \mathrm{~s}$ and
$\nu_{m}<\nu_{\mathrm{c}}<\nu_{\mathrm{LAT}}$ at $\gtrsim 316 \mathrm{~s}$, further implying that $\nu_{\mathrm{c}, \mathrm{f}}^{\mathrm{syn}}$ crosses the LAT band at $\approx 316 \mathrm{~s}$. The value of $p=2.35 \pm 0.37$ for the FS is also consistent with that previously obtained by Laskar et al. (2016) using the X-ray observations for this burst.

\subsection{Constraint on the Parameters from the Afterglow Observations}

\subsubsection{Afterglow Observations}

The Swift XRT (X-ray Telescope) followed up GRB 160509A in a series of (seven) observations tiled on the sky (Kennea et al. 2016). The data in all modes began to be collected from $7.3 \times 10^{3} \mathrm{~s}$ to 20 days after the trigger time. The XRT data used in this analysis are publicly available in the website database. ${ }^{17}$ The XRT flux was extrapolated from 10 $\mathrm{keV}$ to $1 \mathrm{keV}$ with the conversion factor shown in Evans et al. (2010). In the PC mode, the spectral value of the photon index was $\Gamma_{\mathrm{X}}=\beta_{\mathrm{X}}+1=1.97_{-0.07}^{+0.08}$ for a galactic (intrinsic) absorption of $N_{H}=2.12\left(2.7_{-0.07}^{+0.04}\right) \times 10^{22} \mathrm{~cm}^{-2}$.

Laskar et al. (2016) analyzed the entire XRT data set using HEASOFT (v6.18) to fit the spectra. These authors initially using a broken PL and reported two spectral indices of $\Gamma_{X}=\beta_{X}+1=2.01 \pm 0.05 \quad$ and $\Gamma_{X}=\beta_{X}+1=2.12 \pm 0.05$ for the intervals $7.3 \times 10^{3}$ to $3.7 \times 10^{4} \mathrm{~s}$ and $4.3 \times 10^{4}$ to $1.3 \times 10^{6} \mathrm{~s}$, respectively. Supposing that the spectral index did not evolve during the whole interval, they used a PL and reported an X-ray spectral index of $\Gamma_{X}=2.07 \pm 0.04$ for the entire interval. It is worth noting that the values of the spectral index reported in the website database and by Laskar et al. (2016) are consistent.

Magenta data points in Figure 3 show the XRT light curve obtained at $1 \mathrm{keV} .{ }^{18} \mathrm{In}$ accordance with the shape of the X-ray light curve, it is divided in three intervals, labeled II $\left(3.5 \times 10^{3} \lesssim t \lesssim 5.1 \times 10^{4} \mathrm{~s}\right)$,

III $\left(5.1 \times 10^{4} \underset{\lesssim}{\approx} \lesssim 4.28 \times 10^{5} \mathrm{~s}\right)$, and IV $\left(4.28 \times 10^{5} \mathrm{~s} \lesssim t\right)$. We model each segment of the light curve with a PL function $\left(t^{-\alpha_{\mathrm{X}}}\right)$ using the chi-squared minimization method. The best-fit

\footnotetext{
${ }^{17}$ http://www.swift.ac.uk/xrtproducts/

18 https://www.swift.ac.uk/burst_analyser/00020607/
} 
Table 2

Best-fit Values Found from the XRT Light Curve of GRB 160509A

\begin{tabular}{lccc}
\hline \hline X-rays & $\begin{array}{c}\text { Time interval } \\
(\mathrm{s})\end{array}$ & $\begin{array}{c}\text { Index } \\
\left(\alpha_{\mathrm{X}}\right)\end{array}$ & $\chi^{2} / \mathrm{ndf}$ \\
\hline II & $(0.35-5.1) \times 10^{4}$ & $0.50 \pm 0.12$ & 1.22 \\
\hline III & $(0.51-4.28) \times 10^{5}$ & $1.23 \pm 0.13$ & 1.11 \\
\hline IV & $\geqslant 4.28 \times 10^{5}$ & $2.06 \pm 0.3$ & 1.51 \\
\hline
\end{tabular}

indices are $\alpha_{\mathrm{X}, \mathrm{II}}=0.50 \pm 0.12, \alpha_{\mathrm{X}, \mathrm{III}}=1.23 \pm 0.13$, and $\alpha_{X, I V}=2.06 \pm 0.3$, for intervals II, III, and IV, respectively. The best-fit value of each interval and its corresponding chisquared test statistic are reported in Table 2.

Using the closure relations, the flux during the interval III is described by $F_{\nu, f}^{\text {syn }} \propto t^{-1.23 \pm 0.13} \nu^{-1.07 \pm 0.04}$, which can be understood as FS synchrotron emission in the regime, $\nu_{\mathrm{c}, \mathrm{f}}^{\mathrm{syn}}<\nu$ with $p=2.30 \pm 0.15$. Given that the spectral index remains unchanged during intervals II and IV, the index $\alpha_{\mathrm{X}, \mathrm{II}}=0.50 \pm 0.12$ during interval III is consistent with the plateau phase, while the steepening of the light curve in interval IV $\left(\alpha_{\mathrm{X}, \mathrm{IV}}=2.06 \pm 0.3\right)$ is consistent with post-jet-break evolution.

Our observation that $\nu_{\mathrm{c}, \mathrm{f}}^{\mathrm{syn}}<\nu_{\mathrm{X}}$ at $\gtrsim 5.1 \times 10^{4} \mathrm{~s}$, together with our prior inference that $\nu_{\mathrm{c}, \mathrm{f}}^{\mathrm{syn}} \approx 100 \mathrm{MeV}$ at $\approx 300 \mathrm{~s}$ (Section 3.1.2), implies a rapid evolution of the FS synchrotron cooling frequency, ${ }^{19}$ at least as fast as $\nu_{\mathrm{c}, \mathrm{f}}^{\mathrm{syn}} \propto t^{-2.2}$. This is at variance with the expectation of $\nu_{\mathrm{c}, \mathrm{f}}^{\mathrm{syn}} \propto t^{-1 / 2}$ in a uniformdensity environment (Sari et al. 1998). Similar rapid evolution of $\nu_{\mathrm{c}, \mathrm{f}}^{\mathrm{syn}}$ has been inferred in other cases, with potential explanations involving time evolution of the microphysical parameters, steep circumburst density profiles, energy injection into the FS, and the proximity of the jet break (Racusin et al. 2008; Filgas et al. 2011; van Eerten \& MacFadyen 2012).

Here, we consider the evolution of microphysical parameters $\left(\epsilon_{\mathrm{e}_{\mathrm{f}}} \propto t^{\alpha_{\mathrm{e}}}, \epsilon_{\mathrm{B}_{\mathrm{f}}} \propto t^{\alpha_{\mathrm{B}}}\right)$ as a possible explanation. The total energy transferred from protons to electrons and magnetic field is not well-understood during the FS, so the fraction of electron and the magnetic density during the afterglow could vary (e.g., see Yost et al. 2003; Kumar \& Panaitescu 2003; Ioka et al. 2006; Fan \& Piran 2006; Panaitescu et al. 2006). We emphasize that this is one possible model with the potential to explain the rapid evolution of $\nu_{\mathrm{c}, \mathrm{f}}^{\mathrm{syn}}$. In this model, the synchrotron light curve in the slow-cooling regime is given by

$$
F_{\nu} \propto \begin{cases}t^{\frac{\left(4 \alpha_{e}-3\right)(p-1)+\alpha_{B}(p+1)}{4}} \nu^{-\frac{p-1}{2}} & \nu_{\mathrm{m}, \mathrm{f}}^{\mathrm{syn}}<\nu<\nu_{\mathrm{c}, \mathrm{f}}^{\mathrm{syn}}, \\ t^{\frac{-(3 p-2)+\alpha_{B}(p-2)+4 \alpha_{e}(p-1)}{4}} \nu^{-\frac{p}{2}} & \nu_{\mathrm{c}, \mathrm{f}}^{\mathrm{syn}}<\nu,\end{cases}
$$

where the synchrotron spectral breaks evolve as $\nu_{\mathrm{m}, \mathrm{f}}^{\mathrm{syn}} \propto t^{\frac{-3+4 \alpha_{e}+\alpha_{B}}{2}}$ and $\nu_{\mathrm{c}, \mathrm{f}}^{\mathrm{syn}} \propto t^{\frac{-\left(3 \alpha_{B}+1\right)}{2}}$. It is worth noting that, once the microphysical parameters stop evolving (i.e., $\alpha_{e}=0$ and $\alpha_{B}=0$ ), the standard synchrotron FS model is recovered (Sari et al. 1998).

To summarize, the LAT light curve at $\leqslant 40 \mathrm{~s}$ is consistent with SSC emission from an RS in the thick-shell regime and a uniform-density circumburst environment. The LAT data at $>40 \mathrm{~s}$ and the X-ray light curve are consistent with

\footnotetext{
${ }^{19}$ In fact, $\alpha_{L, 1}$ and $\alpha_{\mathrm{X}, \mathrm{II}}$ are consistent within the error bars, which would suggest that the break in the X-ray light curve at $5.1 \times 10^{4} \mathrm{~s}$ is due to the passage of $\nu_{\mathrm{c}, \mathrm{FS}}^{\mathrm{syn}}$. We verify this in Section 3.3.1.
}

synchrotron emission from the FS in a uniform-density circumburst environment with $p \approx 2.4 \pm 0.1$ and the spectral ordering $\nu_{\mathrm{m}, \mathrm{f}}<\nu_{X}<\nu_{\mathrm{c}, \mathrm{f}}<\nu_{\mathrm{LAT}}$ for $300 \lesssim t \lesssim 5.1 \times 10^{4} \mathrm{~s}$ and $\nu_{\mathrm{m}, \mathrm{f}}<\nu_{\mathrm{c}, \mathrm{f}}<\nu_{X}$ for $t \gtrsim 4.5 \times 10^{4} \mathrm{~s}$.

\subsubsection{Constraint on the Parameters}

We normalize the synchrotron emission at $h \nu=100 \mathrm{MeV}$ and $1 \mathrm{keV}$ for the LAT and X-ray observations, respectively. The synchrotron light curves that evolve in a uniform-density medium before (Sari et al. 1998) and after (Sari et al. 1999) the jet break were used to describe the long-lasting LAT and X-ray emissions, and the $\mathrm{GeV}$ flares observed before $\leqslant 40 \mathrm{~s}$ are fitted with the SSC emission in the thick-shell regime (Equations (7) and (11)). The synchrotron FS model with varying microphysical parameters is used to describe the X-ray flux during the time interval $10^{3}-5.1 \times 10^{4} \mathrm{~s}$ (Equation (21)). The luminosity distance was estimated using the cosmological parameters reported in Planck Collaboration et al. (2020). Using our estimate of $E_{\mathrm{K}}=6.977_{-0.802}^{+0.801} \times 10^{53} \mathrm{erg}$ and the isotropic-equivalent $\gamma$-ray energy, $E_{\gamma, \text { iso }}=(10 \pm 1) \times 10^{52}$ erg (Ajello et al. 2019), we obtain a prompt gamma-ray efficiency of $\eta=0.15_{-0.02}^{+0.02}$.

Our analytical afterglow model, as described in Section 2, is completely determined by a set of nine parameters $\Sigma_{\sigma}=\left\{E_{\mathrm{K}}, n, p, \epsilon_{\mathrm{B}_{\mathrm{f}}}, \epsilon_{\mathrm{B}_{\mathrm{r}}}, \epsilon_{\mathrm{e}_{\mathrm{f}}}, \epsilon_{\mathrm{e}_{\mathrm{r}}}, \alpha_{e}, \alpha_{B}\right\}$. We then assign prior distributions to these parameters for application in a Markov Chan Monte Carlo (MCMC) simulation. Shape-wise, we choose normal distributions for each physical parameter of the system; this allows us to pass the minimum amount of information (and consequently bias) necessary for the simulation. After determining the shape, we then assign a mean and standard deviation for each parameter. The choice of values is such that our prior distributions cover a range of the typical values found for these parameters in the literature of GRB afterglow modeling (e.g., see Kumar \& Barniol Duran 2009; Kumar \& Zhang 2015; Ajello et al. 2019), while maintaining a reasonable computational time. We then assign a likelihood function described by a normal distribution whose mean is our afterglow model and standard deviation is a hyperparameter $\sigma$. For this hyperparameter, we chose a value that returns a distribution sufficiently large that the likelihood can explore the region around the detections containing the data uncertainty, similar to the models we used in Fraija et al. (2019d). We opted to use a half-normal distribution, with static standard deviation, to describe this $\sigma$ parameter; this way, our likelihood is allowed to better explore the space around the observed data, giving more leeway to the sampler. We use the No-U-TurnSampler from the PyMC3 python distribution (Salvatier et al. 2016) to generate a total of 17,300 samples, allowing a total of 7000 tuning iterations. The results of the MCMC analysis are summarized in Table 3. The best-fitting curves are shown in Figure 3. Figure 4 shows a corner plot displaying the onedimensional marginalized posterior distributions for each parameter and the two-dimensional marginalized posterior distributions for each pair of parameters. It is worth nothing that a Bayesian technique of checking the ability of the model to explain the observed data are posterior predictive checks (Gabry et al. 2019). Concerns about the convergence of affineinvariant ensemble samplers in high dimensions are described by Huijser et al. (2015). We use Gelman and Rubin's convergence diagnostic (Gelman \& Rubin 1992) through the parameter $\hat{R}$ for each variable verify the convergence of the 
Table 3

Median Values of Parameters of GRB 160509A Found with Symmetrical Quantiles

\begin{tabular}{lcc}
\hline \hline Parameters & Median & $\hat{R}$ \\
\hline$E_{\mathrm{K}}\left(10^{53} \mathrm{erg}\right)$ & $6.977_{-0.802}^{+0.801}$ & 1.000 \\
$n\left(10^{-4} \mathrm{~cm}^{-3}\right)$ & $4.554_{-1.121}^{+1.128}$ & 0.999 \\
$p$ & $2.400_{-0.071}^{+0.079}$ & 1.000 \\
$\epsilon_{\mathrm{B}_{\mathrm{f}}}\left(10^{-5}\right)$ & $4.105_{-0.918}^{+0.938}$ & 0.999 \\
$\epsilon_{\mathrm{B}_{\mathrm{r}}}\left(10^{-1}\right)$ & $1.100_{-0.100}^{+0.099}$ & 0.999 \\
$\epsilon_{\mathrm{e}_{\mathrm{f}}}\left(10^{-2}\right)$ & $3.101_{-0.305}^{+0.305}$ & 0.999 \\
$\epsilon_{\mathrm{e}_{\mathrm{r}}}\left(10^{-1}\right)$ & $8.000_{-0.079}^{+0.080}$ & 0.999 \\
$\alpha_{e}\left(10^{-1}\right)$ & $-3.199_{-0.0375}^{+0.366}$ & 1.000 \\
$\alpha_{B}$ & $1.401_{-0.142}^{+0.136}$ & 0.999 \\
\hline
\end{tabular}

Note. The external-shock model is used to constrain the values of the parameters.

sampler. For all variables, $\hat{R}$ returned values $\approx 1$, indicating a well-behaved, converging sampler.

Based on the values reported in Table 3, the implications of the results are discussed in the following section.

\subsection{Implications of the Results and Discussion}

\subsubsection{Microphysical Parameters}

Given the microphysical parameter associated with the magnetic field in the RS region $\left(\epsilon_{\mathrm{B}_{\mathrm{r}}}=1.100_{-0.100}^{+0.099} \times 10^{-1}\right)$, the magnetization parameter (see Figure 6 in Zhang \& Kobayashi 2005) lies in the range $0.04 \lesssim \sigma \lesssim 0.2$, which corresponds to a regime for which the RS is produced. In the RS region, the self-absorption, the characteristic, and the cutoff frequency breaks of synchrotron radiation at $30 \mathrm{~s}$ are $3.5 \times 10^{5} \mathrm{~Hz}$, $5.3 \times 10^{14} \mathrm{~Hz}$, and $1.7 \times 10^{16} \mathrm{~Hz}$, respectively. This shows that the synchrotron emission evolves in the slow-cooling regime and lies in the weak self-absorption regime, so a thermal component is not expected by this mechanism (Kobayashi \& Zhang 2003).

The best-fit values of the magnetic field parameters from the FS and RS regions are different. The evolution of the RS requires that the outflow is moderately magnetized $\mathcal{R}_{\mathrm{B}} \approx 35$. $^{20}$

In the radio bands, Laskar et al. (2016) presented a multifrequency radio detection with the VLA beginning 0.36 days after the trigger time. The VLA observations were carried out at frequencies spanning 1.3 and $37 \mathrm{GHz}$. These authors found that the $\mathrm{X}$-ray and radio emission originated from distinct regions. They modeled the radio to X-ray emission as a combination of synchrotron radiation from both the RS and FS. They showed that the radio observations were dominated up to 10.03 days by synchrotron from the RS region. Our best-fit values using LAT and X-ray observations, $E_{\mathrm{K}}=\left(6.977_{-0.802}^{+0.801}\right) \times 10^{53} \mathrm{erg}$,

$n=\left(4.554_{-1.121}^{+1.128}\right) \times 10^{-4} \mathrm{~cm}^{-3}$, and $p=2.400_{-0.081}^{+0.079}$ are consistent with the values of Laskar et al. (2016), who found $E_{\mathrm{K}}=18.7_{-2.6}^{+5.4} \times 10^{52} \mathrm{erg}, \quad n=(8.6 \pm 2.2) \times 10^{-4}, \quad$ and $p=2.39 \pm 0.03$. Our values of the FS microphysical parameters $\quad\left(\epsilon_{\mathrm{B}_{\mathrm{f}}}=4.105_{-0.918}^{+0.938} \times 10^{-5}\right.$ and $\left.\epsilon_{\mathrm{e}_{\mathrm{f}}}=3.101_{-0.305}^{+0.305} \times 10^{-2}\right)$ are much smaller than reported by Laskar et al. (2016) $\left(\epsilon_{\mathrm{B}_{\mathrm{f}}}=0.11_{-0.05}^{+0.07}\right.$ and $\left.\epsilon_{\mathrm{e}_{\mathrm{f}}}=0.84_{-0.08}^{+0.06}\right)$; however, we note that both sets of analyses suffer from some

$\overline{{ }^{20} \text { Laskar et al. (2016) reported a value of } \mathcal{R}_{\mathrm{B}}} \approx 3$. degeneracy, since (i) the observations do not allow us to locate the FS synchrotron self-absorption break, and (ii) $\nu_{\mathrm{m}, \mathrm{FS}}^{\mathrm{syn}}$ is not uniquely constrained, due to the large optical extinction along the line of sight. Thus, it is possible that the true values of these parameters are intermediate between those derived here and in Laskar et al. (2016).

During the plateau phase $\left(10^{3}-5.1 \times 10^{4} \mathrm{~s}\right)$, the microphysical parameters are given by $\epsilon_{\mathrm{e}_{\mathrm{f}}}\left(\frac{t}{t_{\mathrm{k}}}\right)^{\alpha_{e}}$ and $\epsilon_{\mathrm{B}_{\mathrm{f}}}\left(\frac{t}{t_{\mathrm{k}}}\right)^{\alpha_{\mathrm{B}}}$, with best-fit values of $\alpha_{e}=-3.199_{-0.375}^{+0.366} \times 10^{-1}$ and $\alpha_{B}=1.401_{-0.142}^{+0.136}$ for a normalization time fixed to $t_{\mathrm{k}}=10^{3} \mathrm{~s}$. While the magnetic parameter increases with time, the electron density parameter decreases. It is worth noting that different authors have considered distinct possibilities in the evolution of the microphysical parameters (e.g., see Fan \& Piran 2006; Panaitescu et al. 2006; Kumar \& Panaitescu 2003; Ioka et al. 2006). With the best-fit values, the synchrotron break frequencies evolve as $\nu_{\mathrm{m}, \mathrm{f}}^{\mathrm{syn}} \propto t^{-1.44 \pm 0.05}$ and $\nu_{\mathrm{c}, \mathrm{f}}^{\mathrm{syn}} \propto t^{-2.60 \pm 0.21}$, and the flux as $t^{-0.31 \pm 0.08}$ for $\nu_{\mathrm{m}, \mathrm{f}}^{\mathrm{syn}}<\nu<\nu_{\mathrm{c}, \mathrm{f}}^{\mathrm{syn}}$. One can see that the best-fit value of the temporal index in the interval II (the plateau phase) reported in Table 2 agrees with the predicted value of $-0.31 \pm 0.08$ derived when the microphysical parameters evolve with time. Similarly, the rapid evolution of $\nu_{\mathrm{c}, \mathrm{f}}^{\text {syn }} \propto t^{-2.60 \pm 0.21}$ indicates that the breaks observed in the LAT and the X-ray light curve at $\sim 316 \mathrm{~s}$ and $5.1 \times 10^{4} \mathrm{~s}$, respectively, can be interpreted as the passage of the synchrotron cooling break through the Fermi-LAT and SwiftXRT bands at $100 \mathrm{MeV}$ and $1 \mathrm{keV}$, respectively.

\subsubsection{The Bulk Lorentz Factors}

Our analysis of the multiwavelength afterglow observations leads to an initial bulk and critical Lorentz factors of $\Gamma \simeq 600$ and $\Gamma_{\mathrm{c}} \simeq 350$, respectively. ${ }^{21}$ This shows that our RS model evolving in the thick-shell regime is self-consistent.

The break observed in the X-ray observations at $t_{j} \simeq 4.5 \times 10^{5} \mathrm{~s}$ is associated with a jet break. This value leads to a jet opening angle of $\theta_{j} \simeq 8.3^{\circ}$ and a bulk Lorentz factor at the jet-break time of $\Gamma_{\mathrm{j}, \mathrm{br}}=6.9$ (Sari et al. 1999). The jet opening angle obtained is twice the value reported in Laskar et al. (2016), so their result is not based solely on the X-ray light curve

The value of the initial bulk Lorentz factor inferred during the deceleration phase is similar to those reported by other burst detected by Fermi-LAT (Veres \& Mészáros 2012). Since GRB 160509A exhibited one of the most energetic photons reported by Fermi-LAT in the second GRB catalog, it is expected that the value of the bulk Lorentz factor lies in the range of the brightest LAT-detected bursts $(500 \lesssim \Gamma \lesssim 1000 ;$ Abdo et al. 2009a; Ackermann 2010; Ackermann et al. 2011; Ackermann 2013; Ackermann et al. 2014; Fraija et al. 2019b, 2019c), as found in this work.

\subsubsection{The Ambient Density Profile}

If we consider the core-collapse scenario for long GRBs (e.g., Woosley 1993; MacFadyen \& Woosley 1999; Woosley \& Bloom 2006) then stellar winds from the massive star, which are expected to form the circumburst medium, scale as a function of the radius (e.g., Chevalier \& Li 1999). Beyond the

\footnotetext{
21 The bulk Lorentz factor is obtained using Equation (2) and the best-fit values reported in Table 3. As expected, the bulk Lorentz factor is above the critical one.
} 


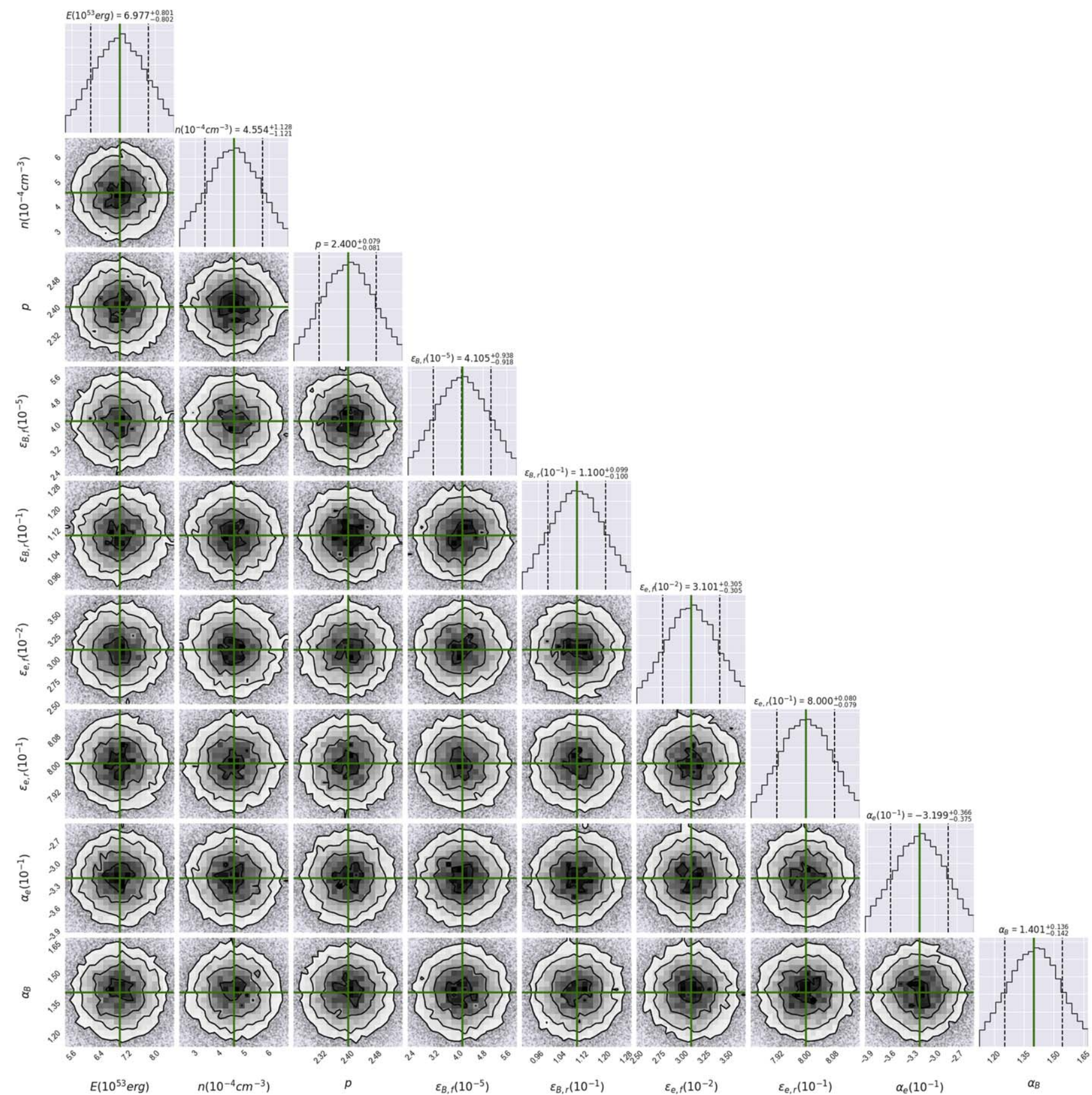

Figure 4. Corner plot of the parameters derived from fitting the LAT and X-ray light curves of GRB 160509A with an RS-SSC and FS synchrotron model, together with median values (green lines) and $1 \sigma$ credible intervals (dashed lines). MCMC summary statistics for all parameters are listed in Table 3 . A set of normal distributions are made for the priors. Values of the mean and standard deviation for each of these normal distributions used for the priors are: $E_{\mathrm{K}}\left(10^{53} \mathrm{erg}\right)=[8.0 ; 1.0], \quad n\left(10^{-4} \mathrm{~cm}^{-3}\right)=[10.0 ; 1.0], \quad p=[2.2 ; 0.1], \quad \epsilon_{\mathrm{B}_{\mathrm{f}}}\left(10^{-5}\right)=[1.0 ; 0.1], \quad \epsilon_{\mathrm{Br}_{\mathrm{r}}}\left(10^{-1}\right)=[1.0 ; 0.1], \quad \epsilon_{\mathrm{e}_{\mathrm{f}}}\left(10^{-2}\right)=[10.0 ; 1.0]$, $\epsilon_{\mathrm{e}_{\mathrm{r}}}\left(10^{-1}\right)=[1.0 ; 0.1], \alpha_{e}\left(10^{-1}\right)=[-3.0 ; 0.1]$ and $\alpha_{B}=[1.0 ; 0.1]$.

wind termination shock, the density profile is expected to transition from wind-like to the uniform-density interstellar medium (Fraija et al. 2017).

Tak et al. (2019) analyzed 26 long bright GRBs and showed that a subset of these events (22 GRBs) could be explained with the uniform-density medium. Other observational studies reached similar conclusions (e.g., Yost et al. 2003; Schulze et al. 2011). These outcomes may imply that a wind profile cannot be explained when the radius of the FS is reached (Schulze et al. 2011).

The best-fit value of the circumburst density $n=4.554_{-1.121}^{+1.128} \times 10^{-4} \mathrm{~cm}^{-3}$ indicates that GRB 160509A exploded in an environment with very low density, comparable to that of a halo or intergalactic medium, with $n \sim 10^{-5}-10^{-3} \mathrm{~cm}^{-3}$. The inferred low density agrees with our prediction (Section 2.3) that the simultaneous occurrence of a GeV flare and a late-time break in the LAT light curve (due to 
the passage of $\nu_{c}^{\text {syn }}$ ) requires $\mathrm{n} \lesssim 10^{-3} \mathrm{~cm}^{-3}$ for typical parameters. A large fraction (22) of LAT-detected GRBs have been shown to have exploded in an environment best described as a uniform-density medium (Tak et al. 2019), and GRB 160509A continues this trend.

Although Laskar et al. (2016) favored a uniform-density environment for this burst, on physical grounds (based on the inferred initial Lorentz factor from the RS emission), they could not conclusively distinguish between a wind and a uniform-density medium based on X-ray, optical, and radio afterglow observations alone. As shown in this paper, analysis of the LAT observations suggests that this is consistent with the evolution of the external shocks in a uniform-density medium.

\subsubsection{VHE Photons above the Synchrotron Limit and SSC Emission from $F S$}

Ajello et al. (2019) presented, in the second Fermi-LAT GRB catalog, the bursts with photon energies above $>10 \mathrm{GeV}$. Two such photons were associated with GRB 160509A: the first photon had an energy of $51.9 \mathrm{GeV}$ and arrived at $76.5 \mathrm{~s}$, and the second one had an energy of $41.5 \mathrm{GeV}$ and arrived at $242 \mathrm{~s}$ after the trigger time. Given the best-fit parameters (see Table 3), the maximum energies that photons produced via synchrotron radiation during the evolution of the FS are 6.82 and $4.43 \mathrm{GeV}$ at 76.5 and $242 \mathrm{~s}$, respectively. ${ }^{22}$ Furthermore, RS SSC emission cannot explain the highest-energy photons detected at $76.5 \mathrm{~s}$ and $242 \mathrm{~s}$ after the trigger time because this component is subdominant to the synchrotron FS emission at $\geqslant 40 \mathrm{~s}$. Therefore, the observed high-energy photons require a process distinct from both FS synchrotron and RS SSC emission. We now consider whether SSC emission from the FS could explain these photons (e.g., see Beniamini et al. 2015; Fraija et al. 2019a).

Following Fraija et al. (2019a) and the parameters reported for this burst (see Table 3), the spectral breaks and the maximum flux for SSC emission in the FS can be expressed as

$$
\begin{aligned}
h \nu_{\mathrm{m}, \mathrm{f}}^{\mathrm{ssc}} \simeq & 7.9 \mathrm{GeV}(1+z)^{\frac{5}{4}} \epsilon_{\mathrm{e}_{\mathrm{f}},-2}^{4} \epsilon_{\mathrm{B}_{\mathrm{f}},-5}^{\frac{1}{2}} n_{-4}^{-\frac{1}{4}} E_{\mathrm{K}, 53}^{\frac{3}{4}} t_{2}^{-\frac{9}{4}}, \\
h \nu_{\mathrm{c}, \mathrm{f}}^{\mathrm{ssc}} \simeq & 2.9 \times 10^{11} \mathrm{TeV}(1+z)^{-\frac{3}{4}}\left(1+Y_{\mathrm{f}}\right)^{-4} \epsilon_{\mathrm{B}_{\mathrm{f}},-5}^{-\frac{7}{2}}, \\
& \times n_{-4}^{-\frac{9}{4}} E_{\mathrm{K}, 53}^{-\frac{5}{3}} t_{2}^{-\frac{1}{4}}, \\
F_{\text {max }, \mathrm{f}}^{\mathrm{ssc}} \simeq & 2.8 \times 10^{-8} \mathrm{mJy}(1+z)^{\frac{3}{4}} \epsilon_{\mathrm{B}_{\mathrm{f}},-5}^{\frac{1}{2}} n_{-4}^{\frac{5}{4}} d_{\mathrm{z}, 28}^{-2} E_{\mathrm{K}, 53}^{\frac{5}{4}} \\
& \times t_{2}^{\frac{1}{4}},
\end{aligned}
$$

where $Y_{\mathrm{f}}$ is the Compton parameter for the FS (Wang et al. 2010; Beniamini et al. 2015; Fraija et al. 2019a). In the slowcooling regime the SSC light curve is given by (e.g., Fraija et al. 2019a)

$$
F_{\nu, f}^{\mathrm{ssc}}= \begin{cases}A_{1} t_{2}(h \nu)_{10}^{\frac{1}{3}}, & \nu<\nu_{\mathrm{m}, \mathrm{f}}^{\mathrm{ssc}}, \\ A_{\mathrm{m}} t_{2}^{-1.33}(h \nu)_{10}^{-0.7}, & \nu_{\mathrm{m}, \mathrm{f}}^{\mathrm{ssc}}<\nu<\nu_{\mathrm{c}, \mathrm{f}}^{\mathrm{ssc}}, \\ A_{\mathrm{h}} t_{2}^{-1.45}(h \nu)_{10}^{-1.2}, & \nu_{\mathrm{c}, \mathrm{f}}^{\mathrm{ssc}}<\nu,\end{cases}
$$

\footnotetext{
${ }^{22}$ We use the upper limit on the energy of photons that can be produced by synchrotron radiation in FSs (e.g., see Kumar \& Barniol Duran 2009; Fraija et al. 2019a).
}

where $(h \nu)_{10}=10 \mathrm{GeV}$ and $t_{2}=100 \mathrm{~s}$ correspond to the energy band and timescale of this process, and the coefficients are given by

$$
\begin{aligned}
A_{\mathrm{l}} \simeq & 1.9 \times 10^{-8} \mathrm{mJy}(1+z)^{\frac{1}{3}} \epsilon_{\mathrm{B}_{\mathrm{f}},-5}^{\frac{1}{3}} \epsilon_{e_{f},-2}^{-\frac{4}{3}} n_{-4}^{\frac{4}{3}} d_{\mathrm{z}, 28}^{-2} E_{\mathrm{K}, 53} \\
A_{\mathrm{m}} \simeq & 2.6 \times 10^{-8} \mathrm{mJy}(1+z)^{1.63} \epsilon_{\mathrm{B}_{\mathrm{f}},-5}^{0.85} \epsilon_{e_{f},-2}^{2.8} n_{-4}^{1.08} d_{\mathrm{z}, 28}^{-2} \\
& \times E_{\mathrm{K}, 53}^{1.78}, \\
A_{\mathrm{h}} \simeq & 9.7 \times 10^{-2} \mathrm{mJy}(1+z)^{1.25}\left(1+Y_{\mathrm{f}}\right)^{-2} \epsilon_{\mathrm{B}_{\mathrm{f}},-5}^{-0.45} \epsilon_{e_{f},-2}^{2.8} \\
& \times d_{\mathrm{z}, 28}^{-2} E_{\mathrm{K}, 53}^{1.15} .
\end{aligned}
$$

Given Equations (22) and (23), the two energetic photons at $76.5 \mathrm{~s}$ and $242 \mathrm{~s}$ associated with GRB 160509A lie in the range of $\nu_{\mathrm{m}, \mathrm{f}}^{\mathrm{ssc}}<\nu<\nu_{\mathrm{c}, \mathrm{f}}^{\mathrm{ssc}}$. In this case, the number of VHE photons $\left(N_{\gamma}\right)$ arriving to the LAT effective area $(A)$ at $100 \mathrm{~s}$ can be estimated as $N_{\gamma} \sim 1 \mathrm{ph}\left(\frac{F_{\nu, f}^{\text {ssc }}}{10^{-8} \frac{\text { erg }}{\mathrm{cm}^{2} \mathrm{~s}}}\right)\left(\frac{100 \mathrm{~s}}{t}\right)\left(\frac{10^{4} \mathrm{~cm}^{2}}{A}\right)\left(\frac{h \nu}{10 \mathrm{GeV}}\right)$, which is consistent with observations. The conversion from $1 \mathrm{mJy}$ normalized at $10 \mathrm{GeV}$ to $\simeq 0.2 \mathrm{erg} \mathrm{cm}^{-2} \mathrm{~s}^{-1}$ in the $(0.1-100) \mathrm{GeV}$ energy range for a spectral index of 2 is used.

Taking into account the distance to GRB 160509A, the SSC flux must be corrected by the extragalactic background light (EBL) attenuation. Using the model proposed in Franceschini $\&$ Rodighiero (2017), the attenuation factor $\exp \left\{-\tau\left(\epsilon_{\gamma}, z\right)\right\}$ at $h \nu=100 \mathrm{GeV}$ and $z=1.17$ is $\approx 0.5$.

The frequency above which $\mathrm{KN}$ effects are important can be written as

$$
\begin{aligned}
h \nu_{\mathrm{c}, \mathrm{f}}^{\mathrm{KN}} \simeq & 3.9 \times 10^{3} \mathrm{TeV}(1+z)^{-\frac{3}{4}}\left(1+Y_{\mathrm{f}}\right)^{-1} \epsilon_{\mathrm{B}_{\mathrm{f}},-4}^{-1} n_{-4}^{-\frac{3}{4}} \\
& \times E_{\mathrm{K}, 53}^{-\frac{1}{4}} t_{2}^{-\frac{1}{4}}
\end{aligned}
$$

which is above the energy range considered. Given the minimum $\left(\gamma_{\mathrm{m}}\right)$ and cooling $\left(\gamma_{\mathrm{c}}\right)$ electron Lorentz factors shown in Fraija et al. (2019a), the synchrotron and SSC luminosity ratio can be computed as (Sari \& Esin 2001)

$$
\frac{L_{\nu}^{\mathrm{ssc}}}{L_{\nu}^{\mathrm{syn}}} \simeq 0.7 n_{-4} R_{18} \gamma_{\mathrm{c}, 6}^{2}\left(\frac{\gamma_{\mathrm{c}, 7}}{\gamma_{\mathrm{m}, 3}}\right)^{-1.4},
$$

where $R=9.9 \times 10^{17} \mathrm{~cm}(1+z)^{-\frac{1}{4}} n_{-4}^{-\frac{1}{4}} E_{\mathrm{K}, 53}^{\frac{1}{4}} t_{2}^{\frac{1}{4}}$ is the FS radius. In the case of a uniform medium, $70 \%$ of the synchrotron luminosity is upscattered by SSC emission. The $\mathrm{KN}$ suppression for the SSC photon is important above $\sim 10^{3} \mathrm{TeV}$.

The HAWC observatory performed a search for VHE (0.1-100 TeV) photons in temporal (using four search windows) and spatial coincidence with GRB 160509A (Lennarz \& Taboada 2016). One of these time windows corresponds to around the time (from-20 to $20 \mathrm{~s}$ ) of the highest-energy photon reported by LAT $77 \mathrm{~s}$ after the trigger time. In all the time windows including around the highestenergy photon were consistent with background only.

At the trigger time reported by Fermi-LAT, GRB 160509A was at an elevation of $\theta \approx 27^{\circ} 98$ and culminated at $33^{\circ}$ inside the HAWC's field of view (Lennarz \& Taboada 2016). Taking into account the sensitivity of HAWC to GRBs described in Abeysekara et al. (2012) for a range of the zenith angle between $0.7 \geqslant \cos \theta>0.6$, an upper limit at $100 \mathrm{GeV}$ and a 
spectral index of 2 could be derived as $\approx 1.14 \times 10^{-4} \mathrm{mJy}$. In this case, the theoretical flux predicted through Equation (23) is $\simeq 10^{-11} \mathrm{mJy}$, which agrees with the upper limit derived.

\section{Predicted Light Curve of GRB 180418A: Thin-shell Case}

At 06:44:06.012 UTC on 2018 April 18, the Swift BAT instrument triggered and located GRB 180418A (D'Elia et al. 2018). The BAT light curve in the energy range of $15-150 \mathrm{keV}$ exhibited a single FRED-like pulse with a duration of $T_{90}=1.504 \pm 0.380 \mathrm{~s}$. At 06:44:06.28 UTC on 2018 April 18, Fermi GBM triggered on GRB 180418A (Bissaldi \& Veres 2018). The light curve consisted of a single FRED-like peak similar to the BAT light curve, with a duration of $T_{90}=2.56 \pm 0.20 \mathrm{~s}$ and a fluence of $(5.9 \pm 0.1) \times 10^{-7} \mathrm{erg} \mathrm{cm}^{-2}$ measured in the energy range of $10-1000 \mathrm{keV}$. The Fermi-LAT instrument did not detect GRB 180418A. Because of an observing constraint, the Swift XRT and UVOT instruments could not begin observing this burst until $3081.4 \mathrm{~s}$ and $3086 \mathrm{~s}$ after the trigger time, respectively. The TAROT and RATIR optical telescopes started observing GRB 180418A in several filters 28.0 and $120.6 \mathrm{~s}$ after the trigger time, respectively (Becerra et al. 2019).

Becerra et al. (2019) presented observations of GRB 180418A in $\gamma$-ray, X-ray, and optical bands, suggesting that this burst may have been an sGRB. This burst exhibited a bright optical flare $(\approx 14.2 \mathrm{AB}$ mag in the $r$ band; see Becerra et al. 2019) peaking between 28 and $90 \mathrm{~s}$ after the trigger time. The early optical observations were interpreted as synchrotron RS model in the thin-shell regime and in a uniform-density medium for $p=2.35 \pm 0.03$. Taking into account the isotropic-equivalent kinetic energy of $E_{\mathrm{K}}=7.7 \times 10^{50} \mathrm{erg}$ and a redshift of $z=0.5$, the values of the parameters found by Becerra et al. (2019) are: circumburst density $\left(n=0.15 \mathrm{~cm}^{-3}\right)$, bulk Lorentz factor $(\Gamma=160)$, and microphysical parameters $\left(\epsilon_{\mathrm{e}_{\mathrm{r}}}=0.1\right.$ and $\left.\epsilon_{\mathrm{B}_{\mathrm{r}}}=0.2\right)$.

Using Equations (15) and (19) and the parameters reported by Becerra et al. (2019), we plot the theoretically predicted SSC emission from the RS evolving in the thin-shell regime and the Fermi-LAT sensitivity extrapolated at $1 \mathrm{GeV}$, as shown in Figure 5. This figure illustrates the X-ray and optical observations with the best-fit curve for synchrotron RS emission $^{23}$ and the predicted SSC emission. ${ }^{24}$ It shows that the SSC emission is around two orders of magnitude below the Fermi-LAT sensitivity, which agrees with the nondetections reported by this instrument.

\section{Summary}

We have derived the SSC light curves from RS in thick- and thin-shell regimes for a uniform-density medium and shown that this emission in the thick-shell case could describe the $\mathrm{GeV}$ flares exhibited in some interesting LAT-detected bursts (Ghisellini et al. 2010; Lü et al. 2017). Since the shock crossing time is less than the prompt emission in the thick-shell regime, a bright RS SSC peak is expected at the beginning of the FS emission. By contrast, as the shock crossing time is longer than the duration of the prompt emission in the thinshell regime, a bright RS SSC peak, in this case, appears

\footnotetext{
23 The derived quantities, such as the synchrotron break frequencies, fluxes, and the evolution of the Lorentz factor, are reported in Becerra et al. (2019). ${ }^{24}$ The optical peak is well-separated from the prompt emission, which is a hallmark of the thin-shell model.
}

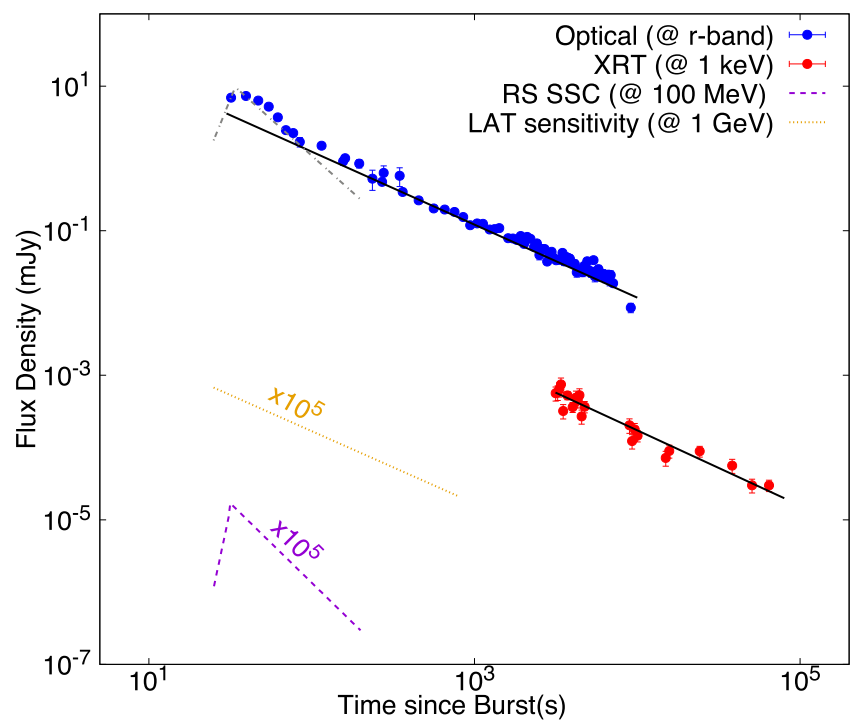

Figure 5. Multiwavelength light curves of GRB 180418A, with best-fit curves. Synchrotron FS model was used to describe X-ray and optical observations. Synchrotron RS model was used to describe the bright optical peak. Theoretically predicted SSC emission in the thin-shell regime and the FermiLAT sensitivity at $1 \mathrm{GeV}$ are displayed in magenta and yellow, respectively. Data points with the fit are taken from Becerra et al. (2019).

distinct from the prompt emission and can be expected during the FS emission. The rise and decay indices of the RS SSC light curves are expected to be $-\frac{p+3}{4} \lesssim \alpha_{\text {ris }} \lesssim-\frac{1}{4}$ and $\frac{29}{96} \lesssim \alpha_{\text {dec }} \lesssim \frac{p+4}{2}$ for a thick-shell regime, and $-\frac{12 p-7}{2} \lesssim \alpha_{\text {ris }} \lesssim-\frac{1}{2}$ and $\frac{6}{35} \lesssim \alpha_{\text {dec }} \lesssim \frac{p+4}{2}$ for a thin-shell regime, respectively. We have shown that a bright RS SSC peak is expected when the microphysical parameter $\epsilon_{\mathrm{e}, \mathrm{r}}$ is above 0.1 and suppressed when $\mathcal{R}_{\mathrm{e}} \ll 1$.

We have also investigated the nature of late-time breaks in $\mathrm{GeV}$ light curves, and interpret these as the passage of the synchrotron cooling frequency of the FS through the $\mathrm{GeV}$ band. This naturally occurs in a uniform, low-density environment. For more energetic bursts, a lower density is needed for this effect to be observed. We have shown that the simultaneous presence of a GeV flare and a break in the LAT light curve requires a low density and $\mathcal{R}_{\mathrm{B}} \gg 1$, suggesting that the outflow could be endowed with primordial magnetic fields in such cases.

We emphasize that the FS closure relations are not expected to be satisfied when the LAT light curves are a superposition of SSC and synchrotron from RS and FS, respectively. Only when the RS SSC emission is suppressed (or has decreased sufficiently such that it is negligible regarding the synchrotron FS emission) can the closure relation be satisfied in the LAT energy band. It is worth highlighting that, depending on the parameter values, $\mathrm{GeV}$ flare RS SSC emission could be hidden by longer-lasting FS synchrotron emission.

As a particular case for the thick-shell regime, we have studied the LAT observations of GRB 160509A, which exhibited a clear, bright peak at $20 \mathrm{~s}$ with a break at $316 \mathrm{~s}$ in the light curve. With the values of the best-fit parameters, we inferred that the first photon with an energy of $51.9 \mathrm{GeV}$ arriving at $76.5 \mathrm{~s}$ and the second one of $41.5 \mathrm{GeV}$ arriving at $242 \mathrm{~s}$ after the trigger time is produced in the deceleration phase of the outflow, and a different mechanism to the standard synchrotron model has to be invoked to interpret these VHE 
photons. We explicitly showed that the SSC FS emission generates these VHE photons. The best-fit values of the microphysical parameters indicate that a magnetized outflow could explain the features exhibited in the light curves of GRB 160509A.

As an example of the thin-shell regime, we have predicted the light curves at $100 \mathrm{MeV}$ for GRB 180418A with the parameters used to describe the X-ray and optical observations. As expected, the light curves at $100 \mathrm{MeV}$ are below the FermiLAT sensitivity.

We thank Xiang-Yu Wang, Alan Watson and B. B. Zhang for useful discussions and comments. N. F. acknowledges support from UNAM-DGAPA-PAPIIT through grants IA102019 and IN10751. R. B. D. acknowledges support from the National Science Foundation (NSF) under grant 1816694. M. G. D. acknowledges support from the American Astronomical Society Chretienne Fellowship and from MINIATURA2 grant 2018/02/X/ST9/03673.

\section{ORCID iDs}

N. Fraija (ㄱ) https://orcid.org/0000-0002-0173-6453

T. Laskar (1) https://orcid.org/0000-0003-1792-2338

S. Dichiara (1) https://orcid.org/0000-0001-6849-1270

P. Beniamini (1) https://orcid.org/0000-0001-7833-1043

M. G. Dainotti (1) https://orcid.org/0000-0003-4442-8546

R. L. Becerra (i) https://orcid.org/0000-0002-0216-3415

\section{References}

Abdo, A. A., Ackermann, M., Ajello, M., et al. 2009a, ApJL, 706, L138 Abdo, A. A., Ackermann, M., Arimoto, M., et al. 2009b, Sci, 323, 1688 Abeysekara, A. U., Aguilar, J. A., Aguilar, S., et al. 2012, APh, 35, 641 Ackermann, M., Asano, K., Atwood, W. B., et al. 2010, ApJ, 716, 1178 Ackermann, M., Ajello, M., Asano, K., et al. 2011, ApJ, 729, 114 Ackermann, M., Ajello, M., Asano, K., et al. 2014, Sci, 343, 42 Ackermann, M., Ajello, M., Asano, K., et al. 2013, ApJ, 763, 71 Ajello, M., Arimoto, M., Axelsson, M., et al. 2019, ApJ, 878, 52 Alexander, K. D., Laskar, T., Berger, E., et al. 2017, ApJ, 848, 69 Ayache, E. H., van Eerten, H. J., \& Daigne, F. 2020, MNRAS, 495, 2979 Becerra, R. L., Dichiara, S., Watson, A. M., et al. 2019, ApJ, 881, 12 Bégué, D., Pe'er, A., \& Lyubarsky, Y. 2017, MNRAS, 467, 2594 Beniamini, P., Barniol Duran, R., \& Giannios, D. 2018, MNRAS, 476, 1785 Beniamini, P., \& Giannios, D. 2017, MNRAS, 468, 3202 Beniamini, P., \& Granot, J. 2016, MNRAS, 459, 3635

Beniamini, P., Nava, L., Duran, R. B., \& Piran, T. 2015, MNRAS, 454, 1073 Beniamini, P., \& Piran, T. 2014, MNRAS, 445, 3892

Beniamini, P., \& van der Horst, A. J. 2017, MNRAS, 472, 3161

Bissaldi, E., \& Veres, P. 2018, GCN, 22656, 1

Brun, R., \& Rademakers, F. 1997, NIMPA, 389, 81

Cavallo, G., \& Rees, M. J. 1978, MNRAS, 183, 359

Chevalier, R. A., \& Li, Z.-Y. 1999, ApJL, 520, L29

Dainotti, M. G., Ostrowski, M., \& Willingale, R. 2011, MNRAS, 418, 2202

D'Elia, V., D'Ai, A., Evans, P. A., et al. 2018, GCN, 22646, 1

de Angelis, A., Tatischeff, V., Tavani, M., et al. 2017, ExA, 44, 25

Evans, P. A., Willingale, R., Osborne, J. P., et al. 2010, A\&A, 519, A102

Fan, Y., \& Piran, T. 2006, MNRAS, 369, 197

Filgas, R., Greiner, J., Schady, P., et al. 2011, A\&A, 535, A57

Fraija, N. 2015, ApJ, 804, 105

Fraija, N., Barniol Duran, R., Dichiara, S., \& Beniamini, P. 2019a, ApJ, 883,162

Fraija, N., Dichiara, S., Pedreira, A. C. C. D. E. S., et al. 2019b, ApJL, 879, L26

Fraija, N., Dichiara, S., \& Pedreira, A. C. C. D. E. S. 2019c, ApJ, 885, 29

Fraija, N., Lee, W., \& Veres, P. 2016a, ApJ, 818, 190
Fraija, N., Lee, W. H., Veres, P., \& Barniol Duran, R. 2016b, ApJ, 831, 22 Fraija, N., Pedreira, A. C. C. D. E. S., \& Veres, P. 2019d, ApJ, 871, 200 Fraija, N., \& Veres, P. 2018, ApJ, 859, 70

Fraija, N., Veres, P., Zhang, B. B., et al. 2017, ApJ, 848, 15

Franceschini, A., \& Rodighiero, G. 2017, A\&A, 603, A34

Gabry, J., Simpson, D., Vehtari, A., Betancourt, M., \& Gelman, A. 2019, J. R. Stat. Soc. Series A, 182, 389

Gelman, A., \& Rubin, D. B. 1992, StaSc, 7, 457

Ghisellini, G., Ghirlanda, G., Nava, L., \& Celotti, A. 2010, MNRAS, 403, 926

Giannios, D. 2008, A\&A, 480, 305

Huijser, D., Goodman, J., \& Brewer, B. J. 2015, arXiv:1509.02230

Ioka, K., Toma, K., Yamazaki, R., \& Nakamura, T. 2006, A\&A, 458, 7

Kennea, J. A., Roegiers, T. G. R., Osborne, J. P., et al. 2016, GCN, 19408, 1

Kobayashi, S. 2000, ApJ, 545, 807

Kobayashi, S., \& Sari, R. 2000, ApJ, 542, 819

Kobayashi, S., \& Zhang, B. 2003, ApJ, 597, 455

Kobayashi, S., \& Zhang, B. 2007, ApJ, 655, 973

Kobayashi, S., Zhang, B., Mészáros, P., \& Burrows, D. 2007, ApJ, 655, 391

Kumar, P., \& Barniol Duran, R. 2009, MNRAS, 400, L75

Kumar, P., \& Barniol Duran, R. 2010, MNRAS, 409, 226

Kumar, P., \& Panaitescu, A. 2000, ApJL, 541, L51

Kumar, P., \& Panaitescu, A. 2003, MNRAS, 346, 905

Kumar, P., \& Zhang, B. 2015, PhR, 561,

Lai, T. L., Robbins, H., \& Wei, C. Z. 1978, PNAS, 75, 3034

Laskar, T., Alexander, K. D., Berger, E., et al. 2016, ApJ, 833, 88

Laskar, T., Berger, E., Zauderer, B. A., et al. 2013, ApJ, 776, 119

Laskar, T., van Eerten, H., Schady, P., et al. 2019, ApJ, 884, 121

Lennarz, D., \& Taboada, I. 2016, GCN, 19423, 1

Longo, F., Bissaldi, E., Bregeon, J., et al. 2016, GCN, 19403, 1

Lü, H., Wang, X., Lu, R., et al. 2017, ApJ, 843, 114

MacFadyen, A. I., \& Woosley, S. E. 1999, ApJ, 524, 262

Maxham, A., Zhang, B.-B., \& Zhang, B. 2011, MNRAS, 415, 77

McKinney, J. C., \& Uzdensky, D. A. 2012, MNRAS, 419, 573

Mészáros, P., \& Rees, M. J. 1997, ApJ, 476, 232

Nousek, J. A., Kouveliotou, C., Grupe, D., et al. 2006, ApJ, 642, 389

Panaitescu, A., \& Kumar, P. 2000, ApJ, 543, 66

Panaitescu, A., Mészáros, P., Burrows, D., et al. 2006, MNRAS, 369, 2059

Panaitescu, A., Vestrand, W. T., \& Woźniak, P. 2014, ApJ, 788, 70

Perley, D. A., Cenko, S. B., Corsi, A., et al. 2014, ApJ, 781, 37

Planck Collaboration, Aghanim, N., Akrami, Y., et al. 2020, A\&A, 641, A6

Piron, F. 2016, CRPhy, 17, 617

Racusin, J. L., Karpov, S. V., Sokolowski, M., et al. 2008, Natur, 455, 183

Roberts, O. J., Fitzpatrick, G., \& Veres, P. 2016, GCN, 19411, 1

Salvatier, J., Wieckiâ, T. V., \& Fonnesbeck, C. 2016, PyMC3: Python Probabilistic Programming Framework, Astrophysics Source Code Library, ascl: 1610.016

Sari, R., \& Esin, A. A. 2001, ApJ, 548, 787

Sari, R., \& Piran, T. 1995, ApJL, 455, L143

Sari, R., \& Piran, T. 1999, ApJL, 517, L109

Sari, R., Piran, T., \& Halpern, J. P. 1999, ApJL, 519, L17

Sari, R., Piran, T., \& Narayan, R. 1998, ApJL, 497, L17

Schulze, S., Klose, S., Björnsson, G., et al. 2011, A\&A, 526, A23

Sironi, L. 2015, ApJ, 800, 89

Tak, D., Omodei, N., Uhm, Z. L., et al. 2019, ApJ, 883, 134

Tam, P.-H. T., He, X.-B., Tang, Q.-W., \& Wang, X.-Y. 2017, ApJL, 844, L7

Tanvir, N. R., Levan, A. J., Cenko, S. B., et al. 2016, GCN, 19419, 1

van Eerten, H. J., \& MacFadyen, A. I. 2012, ApJL, 747, L30

Veres, P., \& Mészáros, P. 2012, ApJ, 755, 12

Vestrand, W. T., Wren, J. A., Wozniak, P. R., et al. 2006, Natur, 442, 172

Wang, X.-Y., He, H.-N., Li, Z., Wu, X.-F., \& Dai, Z.-G. 2010, ApJ, 712, 1232

Willingale, R., Genet, F., Granot, J., \& O’Brien, P. T. 2010, MNRAS, 403, 1296

Willingale, R., O'Brien, P. T., Osborne, J. P., et al. 2007, ApJ, 662, 1093

Woosley, S. E. 1993, ApJ, 405, 273

Woosley, S. E., \& Bloom, J. S. 2006, ARA\&A, 44, 507

Yost, S. A., Harrison, F. A., Sari, R., \& Frail, D. A. 2003, ApJ, 597, 459

Zhang, B., \& Kobayashi, S. 2005, ApJ, 628, 315

Zhang, B., Kobayashi, S., \& Mészáros, P. 2003, ApJ, 595, 950

Zhang, B., \& Mészáros, P. 2004, IJMPA, 19, 2385

Zhang, B., \& Yan, H. 2011, ApJ, 726, 90 